\title{
Research on LHD (Scraper) Path Tracking Control Based on LQR and Predict Pose Information
}

\author{
Yulin Zhang ${ }^{1}$, Chenxi Zhao ${ }^{1}$, Zijian Wang ${ }^{1, *}$ and Haoxuan Yu 1,2, * \\ ${ }^{1}$ School of Resources and Safety Engineering, Central South University, Changsha, Hunan Province, China \\ 8210183024@csu.edu.cn (Y.Z); 8210183019@csu.edu.cn (C.Z) \\ ${ }^{2}$ Institute of Electrical and Electronics Engineers (IEEE), New York City, NY 10001, USA. \\ *Correspondence: 18973155503@163.com (Z.W.); yuhaoxuan@csu.edu.cn (H.Y.)
}

\begin{abstract}
With the depletion of shallow surface resources, the future mining work will develop towards the deep surface, and the objective conditions such as the mining environment will be more complex and dangerous than now, and the requirements for personnel and equipment will be higher and higher. The efficient exploitation of deep space cannot be separated from such mobile and flexible production and transportation equipment as scraper. In the new era, intelligence is the development trend of the LHD, and path tracking control technology is the key to the intelligent LHD, and it is also an urgent problem to be solved for its unmanned driving. This paper describes the realization of the automatic operation function of articulated LHD from two aspects of mathematical model and trajectory tracking control method, and focuses on the research of the path tracking control scheme in the field of unmanned driving, that is, LQR controller. On this basis, the parameters of the LQR controller are optimized by combining different intelligent cluster algorithms to find the optimal solution of the LQR controller.
\end{abstract}

Keywords: LHD (Scraper); Artificial intelligence; Path tracking; The optimal control.

\section{Introduction}

With the development of industry, the global demand for mineral resources continues to increase. At the same time, when the shallow surface resources are increasingly exhausted, the mines will develop in the direction of deep sea, deep ground and deep space in the future. As a kind of large trackless equipment, the scraper plays an important role in the production of underground metal mines, and directly reflects the technical level and production capacity of modern underground mining. For example, the scraper can replace manual work in the deep underground space and other harsh environment, which greatly improves the efficiency of mining.

In recent years, due to the development of excavating equipment and the construction of intelligent mines, the development of scraper is rapid, most mines have begun to apply the more advanced remote control scraper, and has achieved good practical results[1]. Scraper of remote control is not only simple manipulation, safe, and with the advent of the era of 5G, the rapid development of artificial intelligence technology and the popularization and application of robot control technique makes scraper again innovation, further development on the basis of the remote control scraper has humanization service sector, the scraper will study thinking of artificial intelligence, so as to realize autonomous learning self-driving technology. As one of the core problems in the field of unmanned intelligent LHD, the path tracking control technology of the LHD is proposed. 
At present, a control strategy of the LHD is proposed to replace the human brain control with computer control, that is, the LHD can accurately operate according to the instruction when receiving the instruction. This not only ensures the normal underground production and transportation operation, but also the intelligent underground transportation system will greatly improve the production capacity of the mine[3]. However, in order to further promote the development of LHD, the research of path following control still needs a certain theoretical and practical basis[4]. In addition, in order to make the intelligent scraper more widely used in the future, problems such as the influence of high well depth, high temperature and high stress on the operation of the scraper in deep mining need to be solved urgently.

At present, green mine has become the theme of mines in the new era, and the development of green mine is inseparable from the research of intelligent mine, including the continuous innovation of scraper[5]. On the whole, intelligent scraper is the inevitable result of the development of mining equipment. At the same time, the continuous innovation and development of intelligent scrapers will also make great contributions to the construction of green mines.

\section{Mathematical model of underground articulated LHD (scraper)}

In the aspect of mathematical modeling, the kinematics model of the downhole articulated scraper has been widely used in the field of path tracking because of its simple motion mechanism and the ability to obtain accurate model[6].

\subsection{Kinematics model of articulated scraper}

Underground intelligent scraper belongs to articulated car body[7]. The car body is divided into front and rear ends, the front end and the rear end are connected by the hinge point[8]. In the deep tunnel environment, the car body has a small steering radius, so it runs more flexible.

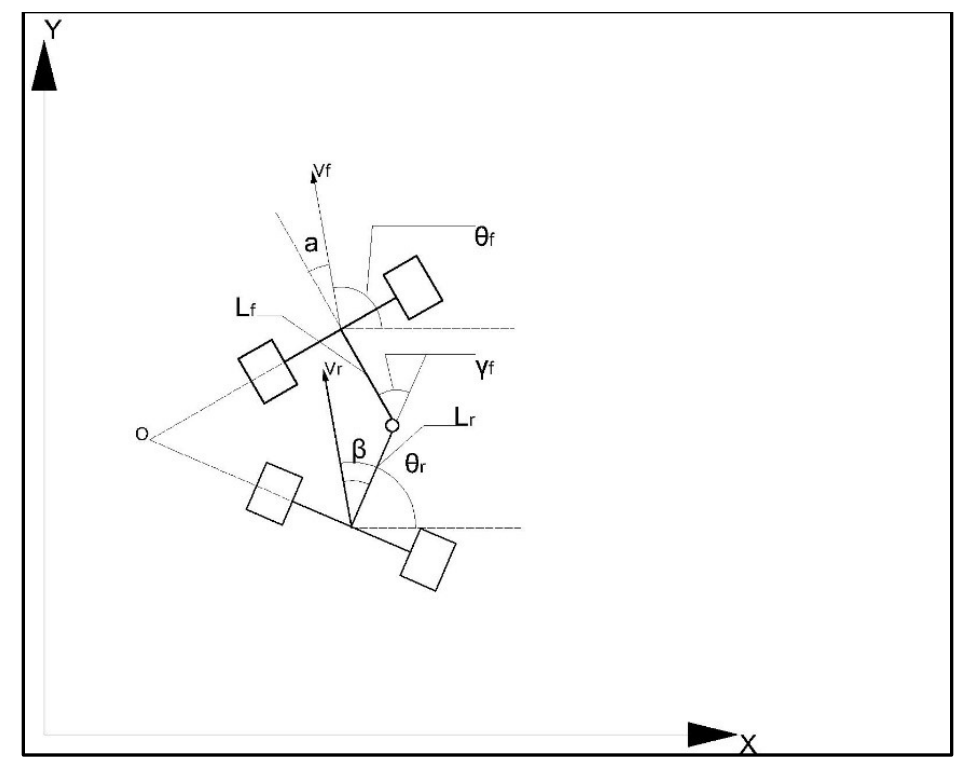

Figure 1. The rotating model 1 of an articulated scraper.

To determine the geometric relationship between the real-time position change information and the motion variables of the LHD, it is necessary to analyze the kinematics of the downhole articulated LHD and establish the kinematics equation[9]. The body structure of the downhole articulated scraper is shown in Figure.1. 
In the coordinate system, the front part of the car body is $\mathrm{P} 1(\mathrm{X} 1, \mathrm{Y} 1)$, the rear part of the car body is $\mathrm{P} 2(\mathrm{X} 2, \mathrm{Y} 2)$; The center of mass velocity of the front body is $\mathrm{v} 1$, and the center of mass velocity of the rear body is v2; The length of the front body is L1, and the length of the rear body is L2; The slip Angle of the front body is $\alpha_{1}$, and the slip Angle of the rear body is $\alpha_{2}$; The heading Angle of the front body is $\theta_{1}$ and the heading Angle of the rear body is $\theta_{2}$; The heading angular velocity of the front body is expressed as, The heading angular velocity of the rear body is expressed as . Define the course angular velocity:

$$
\dot{\gamma}=\dot{\theta}_{1}-\dot{\theta}_{2}
$$

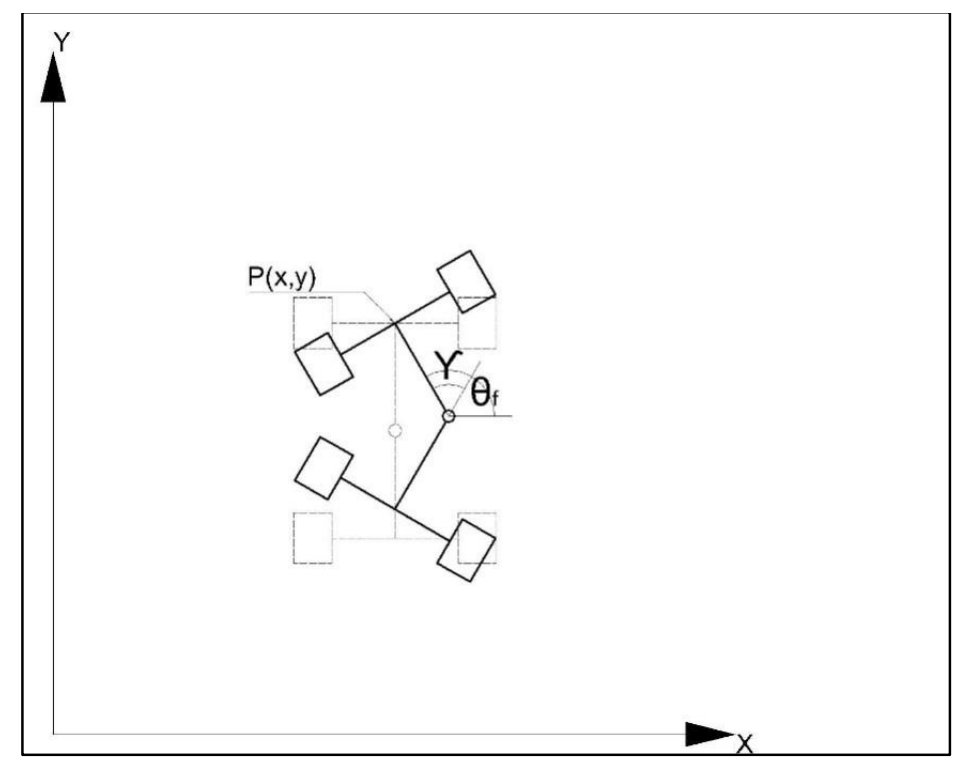

Figure 2. The rotating model 2 of an articulated scraper.

Under normal circumstances, the operation speed of the underground scraper is slow, and generally will not be over $30 \mathrm{~km} / \mathrm{h}$ [10]. If the influence of tire deformation and vehicle body slip is ignored, that is, $\alpha_{1}=\alpha_{2}$, then the motion state model of the down-hole articulated scraper is[11]:

$$
\left\{\begin{array}{c}
-\dot{x}_{1} \sin \theta_{1}+\dot{y}_{1} \cos \theta_{1}=0 \\
-\dot{x}_{2} \sin \left(\theta_{2}+\gamma\right)+\dot{y}_{2} \cos \left(\theta_{2}+\gamma\right) \\
\dot{\theta}_{2}\left(L_{1}+L_{2} \cos \gamma\right)+\dot{\gamma} L_{1}=0 \\
-v_{1}+v_{2} \cos \gamma+\dot{\theta}_{2} L_{2} \sin \gamma=0
\end{array}\right.
$$

Note: $\dot{\gamma}$ is the articulated angular velocity

By selecting the midpoint of the front axle as the reference point of the vehicle state, the kinematics model of the downhole articulated scraper can be obtained as:

$$
\left[\begin{array}{c}
\dot{x} \\
\dot{y} \\
\dot{\theta}_{1} \\
\dot{\gamma}
\end{array}\right]=\left[\begin{array}{cc}
\cos \theta_{1} & 0 \\
\sin \theta_{1} & 0 \\
\frac{\sin _{1}}{L_{1} \cos \gamma+L_{1}} & \frac{L_{1}}{L_{1} \cos \gamma+L_{1}} \\
0 & 1
\end{array}\right]\left[\begin{array}{c}
v_{1} \\
\dot{\gamma}
\end{array}\right]
$$


According to Equation (3), the motion state of the whole vehicle body can be controlled by the articulated angular velocity of the front car body of the downhole articulated scraper[12].

\subsection{Prediction location prediction model}

Prediction location by the current state of movement to solve the motion state of the next moment, the control strategy introduced in predicting the amount of location can be appropriate to control the amount of compensation in advance, to make the error of the controller for the future to make more reasonable control output, avoid the owe control and excessive control, ensure the quality of underground articulated LHD path tracking control[13].

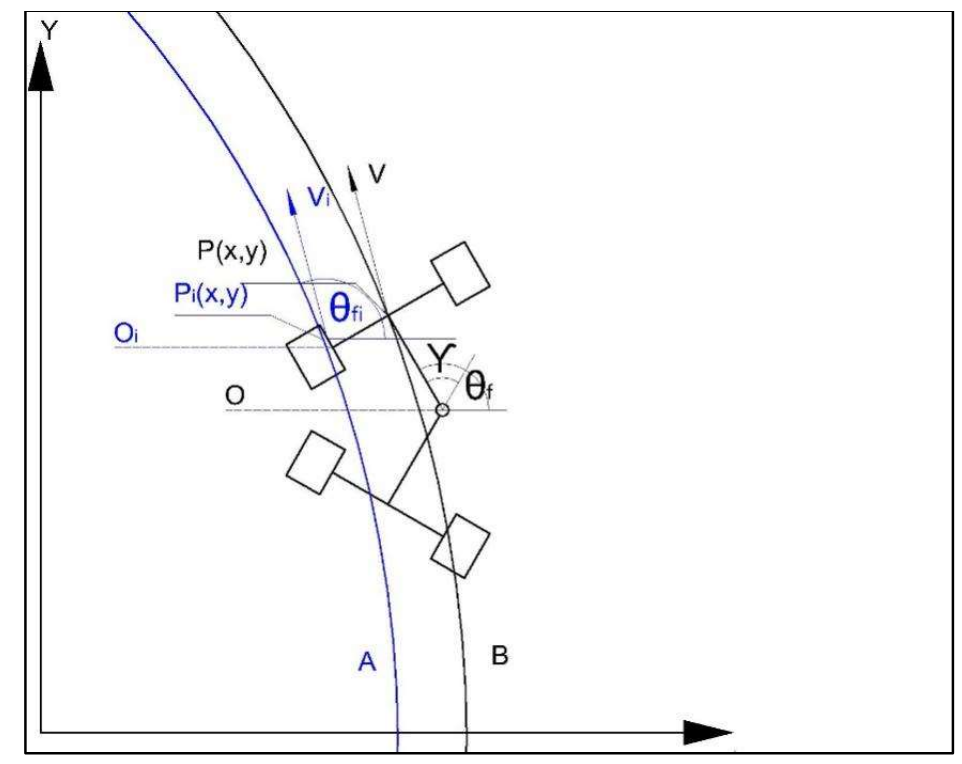

Figure 3. Motion track diagram of the downhole articulated scraper.

Articulated vehicle running curve and parameter definition are shown in Figure.3. Curve $\mathrm{A}$ is the ideal running path of the downhole articulated scraper.

The ideal path of track curve $\mathrm{A}$ and the actual running path of track curve $\mathrm{B}$; $v_{i}$ is the ideal running speed of the articulated scraper, and $v$ is the actual running speed of the articulated scraper; $\theta_{i}$ is the ideal heading Angle of articulated scraper, and $\theta$ is the actual heading Angle of articulated scraper; $P_{i}(x, y)$ is the ideal reference point of the articulated scraper, $P(x, y)$ is the actual reference point of the articulated scraper; $O_{i}$ is the ideal steering center of articulated scraper, and $O$ is the actual steering center of articulated scraper[14].

In addition, the center of the front car body of the downhole articulated scraper is $P$, and the predicted position point of the scraper is $P_{i}$; The steering center of the front car body is $\theta_{1}$, and the steering center of the rear car body is $\theta_{2}$; The steering radius of the front body is $R_{1}$, and the steering radius of the rear body is $R_{2}$.

According to the motion equation of articulated vehicle, the change rate of the front steering Angle of the vehicle is:

$$
\dot{\theta}_{2}=\frac{v_{1} \sin \gamma+L_{2} \dot{\gamma}}{L_{1} \cos \gamma+2}
$$


Set the sampling interval as $\Delta t$, and the predicted heading Angle of the front end of the vehicle is:

$$
\theta_{1}^{\prime}=\theta_{1}+\frac{v_{1} \sin \gamma+L_{2} \dot{\gamma}}{L_{1} \cos \gamma+L_{2}} \Delta t
$$

Assuming that the clockwise rotation direction of the vehicle body is the opposite direction, the solution can be obtained:

$$
R_{1}=\frac{v_{1}\left(L_{1} \cos \gamma+L_{2}\right)}{v_{1} \sin { }_{2} \dot{\gamma}}
$$

In order to distinguish the rotating motion state of the front car body from the linear motion state of the rear car body, the steering state is set as $t_{0}$ and the following conditions are satisfied:

$$
t_{0}=\left\{\begin{array}{lr}
1 & (r>\varepsilon) \\
-1 & (r<-\varepsilon) \\
0 & (-\varepsilon<r<\varepsilon)
\end{array}\right.
$$

Where, the threshold value is selected to be small. When the degree of steering Angle $\gamma$ of the downhole articulated LHD falls within the range of the threshold value, it can be assumed that the LHD does not rotate[15]. When the downhole articulated scraper moves from $P(x, y)$ to $P^{\prime}\left(x^{\prime}, y^{\prime}\right)$, the time taken is $\Delta t$, and the change rate of steering Angle of the front segment of the vehicle is $\mathrm{w}_{1}$, then the Angle of the front segment of the vehicle turns is:

$$
\gamma^{\prime}=\left|w_{1}\right| \Delta t
$$

According to the geometric relation, the forward distance of the vehicle can be calculated:

$$
l=2 R_{1} \sin \frac{\gamma^{\prime}}{2}
$$

It can be deduced that the midpoint $P^{\prime}\left(x^{\prime}, y^{\prime}\right)$ of the front end of the car body in the next period is:

$$
\begin{gathered}
\delta=\left\{\begin{array}{c}
0\left(\theta_{f}=\theta_{f}\right) \\
\theta_{1}+t_{0}\left(\gamma^{\prime} / 2\right)\left(0<\theta_{f}<\pi / 2\right) \\
\pi-\theta_{1}-t_{0}\left(\gamma^{\prime} / 2\right)\left(\pi / 2 \leq \theta_{f} \leq \pi\right) \\
-\theta_{1}-t_{0}\left(\gamma^{\prime} / 2\right)\left(-\pi / 2 \leq \theta_{f} \leq 0\right) \\
\pi+\theta_{1}+t_{0}\left(\gamma^{\prime} / 2\right)\left(-\pi \leq \theta_{f} \leq-\pi / 2\right)
\end{array}\right. \\
y^{\prime}=\left\{\begin{array}{c}
y+v_{1} \Delta t \sin \theta_{1} \\
y+l \sin \delta\left(0<\theta_{f}<\pi / 2\right) \\
y+l \sin \delta\left(\pi / 2 \leq \theta_{f} \leq \pi\right) \\
y-l \sin \delta\left(-\pi / 2 \leq \theta_{f} \leq 0\right) \\
y-l \sin \delta\left(-\pi \leq \theta_{f} \leq-\pi / 2\right)
\end{array}\right. \\
x^{\prime}=\left\{\begin{array}{c}
x+v_{1} \Delta t \cos \theta_{1} \\
x+l \cos \delta\left(0<\theta_{f}<\pi / 2\right) \\
x-l \cos \delta\left(\pi / 2 \leq \theta_{f} \leq \pi\right) \\
x+l \cos \delta\left(-\pi / 2 \leq \theta_{f} \leq 0\right) \\
x-l \cos \delta\left(-\pi \leq \theta_{f} \leq-\pi / 2\right)
\end{array}\right.
\end{gathered}
$$


Note: $\delta$ is the deviation between the predicted heading Angle and the heading Angle of the current position.

\subsection{Subsection}

The core of this paper is to take the speed and hinge Angle of the downhole articulated scraper in the current control scheme as the main control variables, so as to realize the control of the state of the hinge Angle[16]. Based on the predicted heading Angle and the current predicted heading deviation, the control system can effectively improve the control accuracy of reaction path tracking [17]. The average moving speed of the vehicle body is $v$, and the kinematic equation can be calculated according to the error obtained by comparing the actual running path of the downhole articulated scraper with the ideal running path. The error model of the downhole articulated scraper is shown in Figure.4.

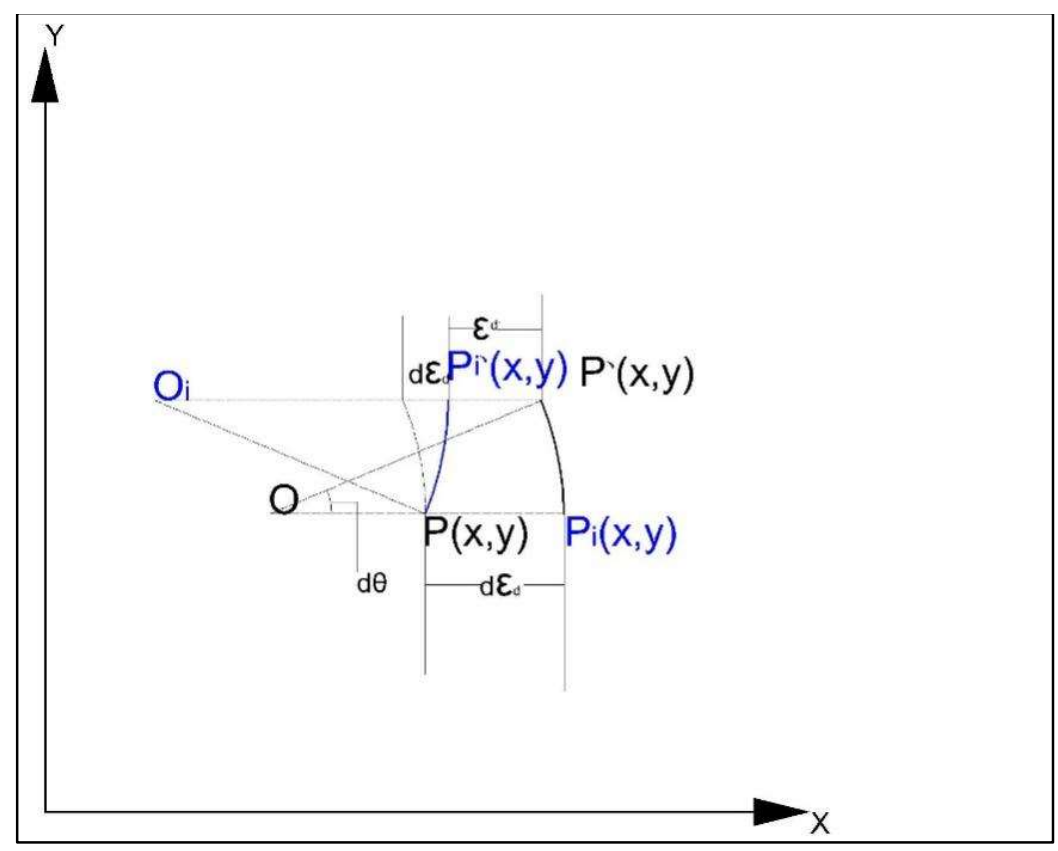

Figure 4. Error dynamic model of articulated scraper.

Position deviation $\delta_{1}$ - the lateral position error between the reference point $P$ of the downhole articulated scraper and the relative point $P$ on the planned path:

$$
\dot{\delta_{1}}=v \delta_{2}
$$

Driving direction deviation $\delta_{0}$ - the difference between the direction Angle of the reference location point $P$ of the downhole articulated scraper and the corresponding direction Angle of the reference location point $P$ on the ideal motion trajectory:

$$
\dot{\delta_{2}}=v \delta_{3}+\dot{r} \frac{L_{2}}{L_{2}+L_{1} \cos r}
$$

Curvature deviation- the curvature error between the reference locus $P$ of the downhole articulated scraper and the reference locus $P$ on the locus:

$$
\dot{\delta}_{3}=\frac{v\left(L_{1}+L_{r} \cos r\right) \dot{r}+L_{2}\left(L_{2}+L_{1} \cos r\right) \ddot{r}+\left(L_{1} L_{2} \sin r\right) \dot{r}^{2}}{v\left(L 2+L_{1} \cos \gamma\right)^{2}}
$$

According to Equations (13) (15) and $L=L_{1}+L_{2}$, it can be obtained: 


$$
\left\{\begin{array}{c}
\dot{\varepsilon}_{1} \\
\dot{\varepsilon}_{2} \\
\dot{\varepsilon_{3}}
\end{array}\right\}=\left[\begin{array}{lll}
0 & v & 0 \\
0 & 0 & v \\
0 & 0 & 0
\end{array}\right]\left\{\begin{array}{c}
\varepsilon_{1} \\
\varepsilon_{2} \\
\varepsilon_{3}
\end{array}\right\}+\left\{\begin{array}{c}
0 \\
0 \\
\left(\frac{1}{L}\right)
\end{array}\right\} \dot{\gamma}+\left\{\begin{array}{c}
0 \\
0 \\
\left(\frac{L_{1}}{v L}\right)
\end{array}\right\} \ddot{\gamma}
$$

Because the underground roadway is narrow, the underground scraper can only run at a low speed when the two sides of the roadway are close to each other[18]. Therefore, the real-time control of the hinged steering Angle of the underground scraper is the key and difficult point in the path tracking process. In order to realize the path tracking of the LHD in the roadway, the steering angular velocity and speed of the articulated Angle were selected as the control variables, and the error dynamics model was established based on the actual path and expected path of the LHD[19]. Articulated vehicle speed is slow, hinge Angle has a small change, and acceleration of hinge Angle can be ignored in general. Therefore, the deviation equation in the above equation is simplified, and the deviation dynamic equation of the downhole articulated scraper is obtained as follows [20]:

$$
\left\{\begin{array}{l}
\dot{\varepsilon}_{1} \\
\dot{\varepsilon}_{2} \\
\dot{\varepsilon_{3}}
\end{array}\right\}=\left[\begin{array}{lll}
0 & v & 0 \\
0 & 0 & v \\
0 & 0 & 0
\end{array}\right]\left\{\begin{array}{l}
\varepsilon_{1} \\
\varepsilon_{2} \\
\varepsilon_{3}
\end{array}\right\}+\left\{\begin{array}{c}
0 \\
\left(\frac{L_{2}}{L}\right) \\
\left(\frac{1}{L}\right)
\end{array}\right\} \dot{\gamma}
$$

The difference between homeopathic heading Angle $\theta_{f}$ and expected heading Angle $\theta_{i}$ was defined as heading Angle deviation $\varepsilon_{\theta}$. The distance between the reference location point $P$ and the expected reference location point $P_{i}$ of the underground scraper is taken as the transverse deviation $\varepsilon_{d}$ (positive when the reference location point $P$ is on the right side of the expected path) : the curvature deviation between the reference location point $P$ and the expected reference location point $P_{i}$ is $\varepsilon_{c}$.

Under the condition of uniform speed, the dynamic model of the deviation of the articulated scraper is a linear time-invariant system model, and the articulated Angle can be controlled by controlling the error variable.

\section{Path Tracking of Underground Articulated LHD Based on LQR Controller}

The control object of LQR optimal controller is a linear system expressed by state space and other basic structures in modern control theory, and all state variables of the system are required to be fully controllable and observable[21]. The core idea of LQR control is to achieve the maximum control effect with the minimum control variable, namely the minimum energy consumption[22]. The optimal state design stage of LQR refers to the $\mathrm{K}$ design of various state feedback microcontrollers required in the optimization stage, which requires that we can simultaneously make the two objective vector functions of quadratic type $\mathrm{Q}$ and $\mathrm{R}$ take the lowest value. The state feedback matrix $\mathrm{K}$ of the target is the unique determination of $Q$ and $R$ matrices in $L Q R$ control, and both $Q$ and $R$ matrices are positive definite matrices[23].

According to the $\mathrm{LQR}$ control theory, the state space equation of the controlled object needs to be determined first. The state equation of the down-hole articulated scraper can be set as the following equations[24]:

$$
\left\{\begin{array}{l}
\dot{X}(t)=A_{1} X(t)+B_{1} u(t) \\
Y(t)=C_{1} X(t)+D_{1} u(t)
\end{array}\right.
$$

Note: $A_{1}$ is the system state space, $A_{1} \in R^{n \times n}$, and $\mathrm{R}$ is the $\mathrm{n}$-dimensional real matrix. $B_{1}$ is the input vector of the system, $B_{1} \in R^{n \times 1}$, and $\mathrm{R}$ is the $\mathrm{n}$-dimensional vector. $C_{1}$ is the system output matrix, $C_{1} \in R^{n \times n}$, and $\mathrm{R}$ is the n-dimensional real matrix. $D_{1}$ is the state feedback vector, $D_{1} \in R^{n \times 1}$, and $\mathrm{R}$ is the n-dimensional real vector. $X(t) 、 u(t)$ 、 $Y(t)$ are system input variables, state variables and outputs. 
According to the above mathematical model of the scraper and vehicle parameters of the articulated scraper in Table1, parameters of the state-space equation of the down-hole articulated scraper can be determined as follows[25]:

$$
A_{1}=\left[\begin{array}{ccc}
0 & 3.5 & 0 \\
0 & 0 & 3.5 \\
0 & 0 & 0
\end{array}\right] \quad B_{1}=\left[\begin{array}{c}
0 \\
\frac{3.44}{5.12} \\
1 \\
5.12
\end{array}\right] \quad C_{1}=\left[\begin{array}{lll}
1 & 0 & 0 \\
0 & 1 & 0 \\
0 & 0 & 1
\end{array}\right]
$$

Table 1. articulated shovel vehicle body parameters

\begin{tabular}{cc}
\hline Parameter Name & Numerical value \\
\hline $\begin{array}{c}\text { Distance from front bridge to articulation } \\
\text { point } \mathbf{L}_{\mathbf{1}} / \mathbf{m}\end{array}$ & 1.766 \\
$\begin{array}{c}\text { Rear bridge distance to articulation point } \\
\text { distance } \mathbf{L}_{\mathbf{2}} / \mathbf{m}\end{array}$ & 1.866 \\
Tire Diameter $\mathbf{d} / \mathbf{m}$ & 1.32 \\
Body width $\mathbf{W} / \mathbf{m}$ & 2.27 \\
Articulated steering angle change range & $0.30 \pi$ \\
$\mathbf{\gamma} / \mathbf{r a d}$ & 7.2 \\
Maximum speed $\mathbf{V}_{\mathbf{m a x}} /\left(\mathbf{m} \times \mathbf{s}^{\mathbf{- 1}}\right)$ & 0.17 \\
Maximum steering angle speed change \\
range $\left.\boldsymbol{\gamma}_{\mathbf{m a x}} / \mathbf{r a d} \times \mathbf{s}^{\mathbf{- 1}}\right)$
\end{tabular}

According to the above matrix parameters, the energy control and visual analysis of the system are:

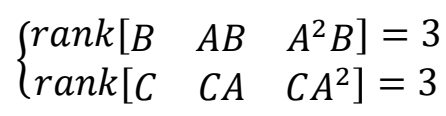

From the above equation, it can be seen that the observability and controllability matrix of the system are full rank, which means that the downhole articulated scraper system is fully controllable and observable, meeting the basic conditions of LQR control[26]. The state space equation model of the system can be determined by the deviation dynamics model and parameters of the downhole articulated LHD:

$$
\left\{\begin{array}{c}
\dot{\varepsilon}(t)=A \varepsilon(t)+B \dot{\gamma}(t) \\
Y(t)=\varepsilon(t)
\end{array}\right.
$$

Through the output deviation matrix $\varepsilon(t)=\left[\begin{array}{lll}\varepsilon_{1} & \varepsilon_{2} & \varepsilon_{3}\end{array}\right]$ of the control system, the ideal articulation Angle input of the truck body can be obtained, and the optimal control performance index can be established[27].

$$
J(\dot{\gamma})=J_{0}(t)+J_{1}(t)=\int_{0}^{\infty}\left[\varepsilon^{T}(t) q \varepsilon(t)+\dot{\gamma}^{T}(t) r \dot{\gamma}(t)\right] d t
$$

Note: $J_{0}$ is the time domain integral of the deviation of the downhole articulated scraper is the error performance index. $J_{1}$ represents the time domain integral of the control quantity, namely the energy consumption index. 
The core of LQR control is to achieve the best control effect with the minimum error performance index and the minimum energy consumption index, establish the feedback control rate $u=-K X(t)$ to achieve $K=R^{-1} B^{T} P$, and establish the Riccati equation:

$$
A^{T} P+P A+Q-P B R^{-1} B^{T} P=0
$$

Note: $P$ is some definite positive matrix, and let $P=E, E$ is the identity matrix.

In order to achieve the best input of the control system, it is necessary to reasonably configure the $Q$ and $R$ matrices to achieve the ideal output of the control quantity[28]. The configuration process of $\mathrm{Q}$ and $\mathrm{R}$ parameters is shown in Figure. 5.

The current deviation

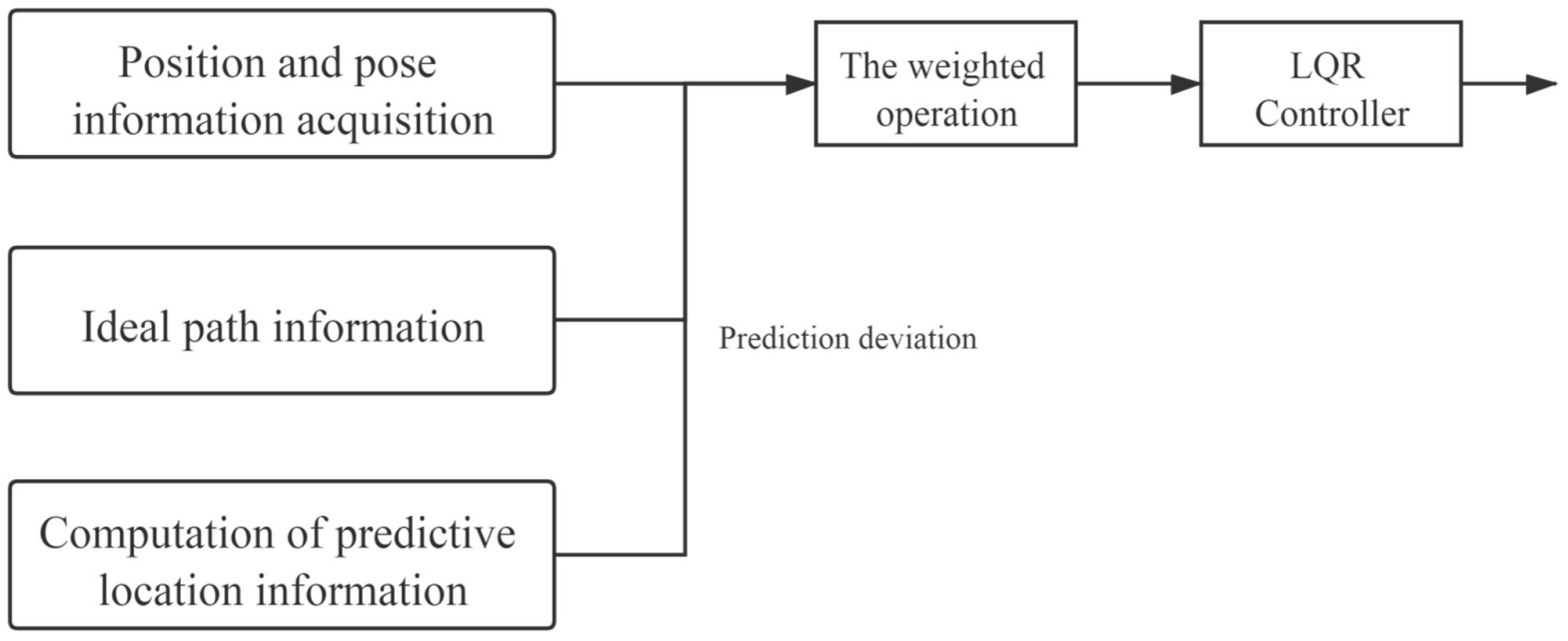

Figure.5 LQR parameter configuration process

The input of LQR controller is composed of three parts. One is the acquisition of the position and posture information of the scraper related to the current position and the input of the LQR controller[29]. Secondly, the acquisition of ideal path information means that the input of LQR controller is related to the position information of ideal path. The third is the collection of predictive position information, which is solved by using the deviation dynamics model[30]. Therefore, the controller input of LQR should be a linear superposition of the above three variables. In order to prevent over-control and under-control of the downhole articulated LHD, different weights should be given to the three variables after comprehensive consideration. The strategy adopted in this paper is to obtain the difference between the current position information and the ideal path, and the predicted position information is to obtain the error by calculating the deviation dynamics model. The final input of the LQR controller is the weighted superposition of the two, as shown in Equation (24).

$$
\left\{\begin{array}{c}
\varepsilon(t)=a \varepsilon_{a}(t)+b \varepsilon_{b}(t) \\
\varepsilon_{a}(t)=\left[\begin{array}{lll}
\varepsilon_{1} & \varepsilon_{2} & \varepsilon_{3}
\end{array}\right] \\
\varepsilon_{b}(t)=\left[\begin{array}{lll}
\varepsilon_{1} & \varepsilon_{2} & \varepsilon_{3}
\end{array}\right]
\end{array}\right.
$$

Note: $\varepsilon_{1}(\mathrm{t})$ is Current Tracking Deviation Matrix; $\varepsilon_{2}(\mathrm{t})$ is Prediction Information Deviation Matrix; $a, b$ is weight factor, $a+b=1 ; \varepsilon(t)$ is the Final input for the LQR controller.

\section{The realization and simulation of intelligent cluster algorithm}

A state variable weight matrix $Q$ in the $L Q R$ control and control the amount of weight matrix $R$, and use that to determine state feedback vector, selection of matrix $Q$ and $R$ 
parameter will directly related to the effects of control, and parameter setting problem in the classical control theory, the selection of two positive definite matrix $Q$ and $R$ tends to rely on a large number of engineering experience, takes longer[31]. Moreover, the optimal parameter configuration of $Q$ and $R$ cannot be obtained. The selection of the weight matrix $\mathrm{Q}$ of the state variable and the weight matrix $\mathrm{R}$ of the control quantity can be simply regarded as the Travel Salesperson Problem. It is difficult to obtain the optimal solution for such problems with general methods, so we need to solve them with the help of some enlightening intelligent clustering algorithms, such as genetic algorithm (GA), Ant Colony Algorithm (ACO), Micro Particle Swarm Optimization (PSO) [32].

\subsection{Adaptive GA algorithm optimization}

4.1.1. Disadvantages of simple genetic algorithms

Simple genetic algorithm (SGA) is of great significance in practical engineering application, but nowadays, many defects of classical simple genetic algorithm are exposed in the process of engineering practice, such as "population precocity", population differentiation and various groups still do not show the identity after various choices and so on[33]. The unreasonable structure of natural selection, crossover and mutation algorithm is the fundamental reason for the precocity problem of the population. The precocity problem cannot be avoided, which is also a major feature of intelligent clustering algorithm. Therefore, it is necessary to improve the crossover operator and mutation operator of the classical genetic algorithm, so as to solve the problem of population precocity to some extent[34].

4.1.2. Improved genetic algorithm-LQR control (LQR-AGA)

In LQR control, as an improved intelligent cluster algorithm, the improved adaptive genetic algorithm overcomes the shortcomings of the traditional LQR control.The parameter selection of $\mathrm{Q}$ and $\mathrm{R}$ is optimized by population, and the crossover mutation operator of the improved genetic algorithm has a strong global optimization ability, and it can find the best state feedback matrix in the selected space[35]. 


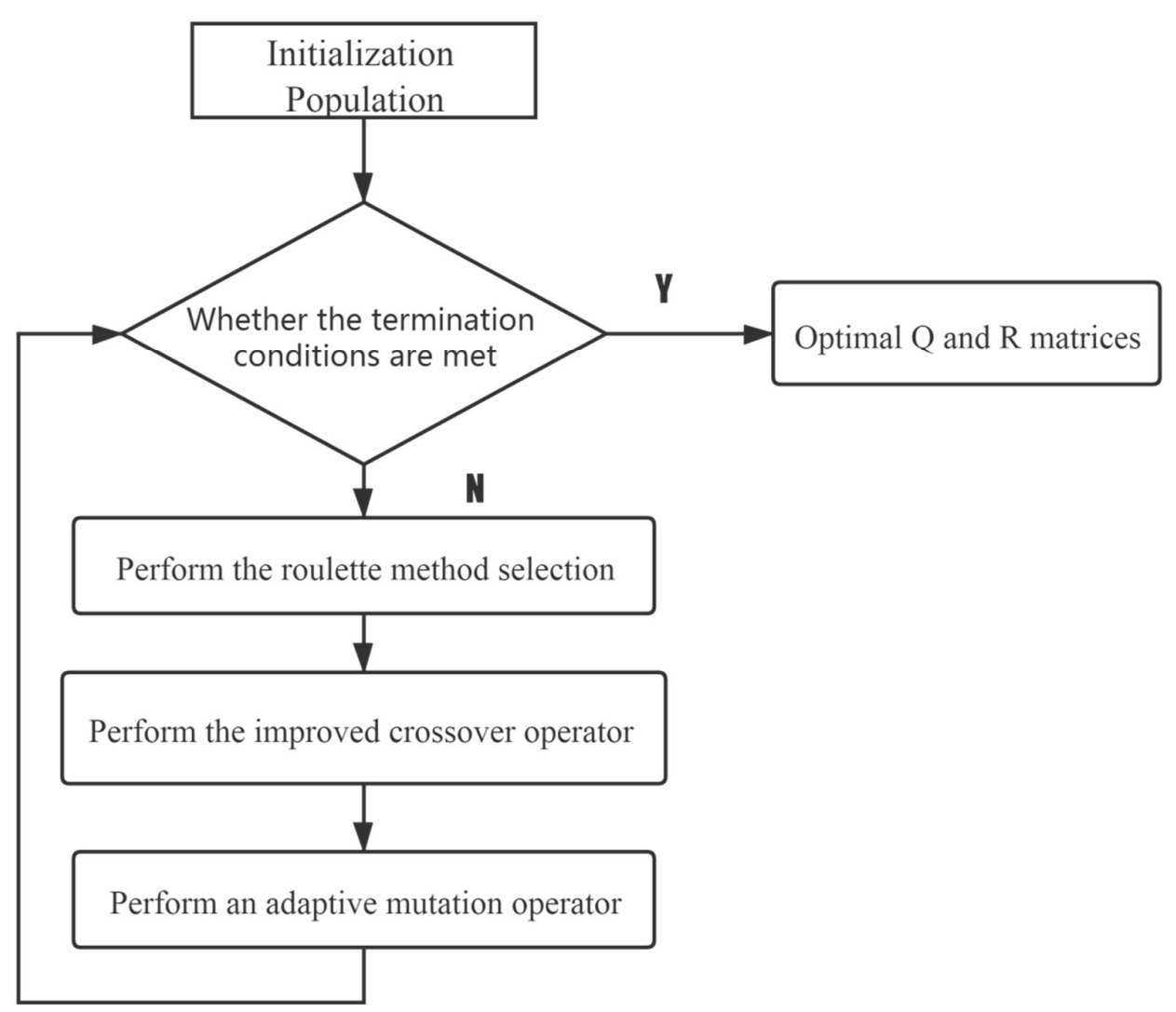

Figure.6 AGA Algorithm Flow Chart

The improved adaptive genetic algorithm operation process is shown in Figure.6, whose main function is to optimize the $\mathrm{Q}, \mathrm{R}$ two matrices in the LQR controllers, that is, to find out the parameters of the optimal $Q=\left[\begin{array}{ccc}q_{1} & 0 & 0 \\ 0 & q_{2} & 0 \\ 0 & 0 & q_{3}\end{array}\right]$ and R matrix.

A. Encoding

The improved adaptive genetic algorithm can encode chromosomes and genes by using the real encoding method because the real encoding method is intuitive, simple and easy to calculate[36]. This method is suitable for the calculation of genetic algorithm with complex fitness function, and can greatly reduce the calculation amount of genetic algorithm, so as to speed up the running efficiency of genetic algorithm[37].

For example, chromosome is assumed to be $Q=\left[\begin{array}{ccc}q_{1} & 0 & 0 \\ 0 & q_{2} & 0 \\ 0 & 0 & q_{3}\end{array}\right]$, of these, $q_{1} q_{2} q_{3}$ are genes on the chromosome, $Q$ is the operation of selection, crossover and mutation operators after the participation of chromosomes and individuals.

B. Group value range

Chromosome $\mathrm{Q}$ is generated according to Matlab random number matrix, that is, the initial trial of the population has a strong randomness, which expands the global optimization ability of the improved adaptive genetic algorithm.

$$
Q=\left[\begin{array}{ccc}
q_{1} & 0 & 0 \\
0 & q_{2} & 0 \\
0 & 0 & q_{3}
\end{array}\right]=\left[\begin{array}{ccc}
50 * \text { rand } & 0 & 0 \\
0 & 50 * \text { rand } & 0 \\
0 & 0 & 50 * \text { rand }
\end{array}\right]
$$


It can be seen from Equation (25) that, $q_{1} \in[0,50], q_{2} \in[0,50], q_{3} \in[0,50]$, the stability of the whole system and the state feedback matrix must exist can be guaranteed by such a value[38].

C. Interleaved mode

The improved crossover operator is used to select the parent generation for crossover change to produce the offspring with strong global search ability.

$$
\begin{aligned}
& \left\{\begin{array}{l}
y_{i}^{(1)}=a\left(x_{i}^{(1)}-x_{i}^{(2)}\right)+b x_{i}^{(1)}+c x_{i}^{(2)} \\
y_{i}^{(2)}=a\left(x_{i}^{(1)}-x_{i}^{(2)}\right)+b x_{i}^{(2)}+c x_{i}^{(1)}
\end{array}\right. \\
& \left\{\begin{array}{l}
y_{i}^{(1)}=b x_{i}^{(1)}+c x_{i}^{(2)} \\
y_{i}^{(2)}=b x_{i}^{(2)}+c x_{i}^{(1)}
\end{array}\right.
\end{aligned}
$$

Note: $x_{i}^{(n)}$ is the $n$ gene above the parent line $i$ chromosome; $y_{i}^{(n)}$ is the $n$ gene above the offspring clause i chromosome; a b c represents the Cross variants.

The two crossover modes are selected according to whether the children cross the boundary or not. If the children cross the boundary, they cross according to Equation (26); otherwise, they cross according to Equation (27) [39].

D. Variation

According to the adaptive mutation operator, the random gene location on the chromosome was mutated to ensure the diversity of the population and enhance its global optimization ability. Meanwhile, it also ensured that the population could have the identity and converge to the optimal solution in the later iteration period.

$$
\Omega=\left\{x_{k}-s(t) \times\left(x_{k}-L_{k}\right), x_{k}+s(t) \times\left(U_{k}-x_{k}\right)\right\}
$$

Note: $s(t)=1-C^{\left[1-\left(\frac{1}{G}\right)\right]^{k}} ; \Omega$ represents Size range of the variation action; $x_{k}$ represents Genes; $L_{k}$ represents Minimum range of variation of the previous generation; $U_{k}$ represents Maximum range of variation of the previous generation; $s(t)$ represents Algebraic variants.

When the number of iterations is small, the gene probability is large and the global optimization ability of the population is strong. When the number of iterations is high, the mutation probability is small, and the computational speed of genetic algorithm is high, and the required time is short[40].

\section{E. Parameter selection}

The number of initialized population individuals was set to be 30 , and 50 generations were bred. The probability of crossover between two chromosomes was 0.2 , the variation action constant $b=3$, and the range of population living space was [0,50].

Through $J(\dot{\gamma})$ discretization of $\mathrm{LQR}$, the fitness equation can be obtained as follows:

$$
F_{(i)}=\sum_{t=1}^{T} q_{1} \varepsilon_{1}{ }^{2}(t)+q_{2}{\varepsilon_{2}}^{2}(t)+q_{2}{\varepsilon_{3}}^{2}(t)+\dot{\gamma}^{2}(t)
$$

Note: $T$ represents total sampling time length; q1, q2, q3 represents $Q$ matrix diagonal element.

To sum up, the block diagram of LQR-AGA control system can be drawn shown as Figure 7[41]. 


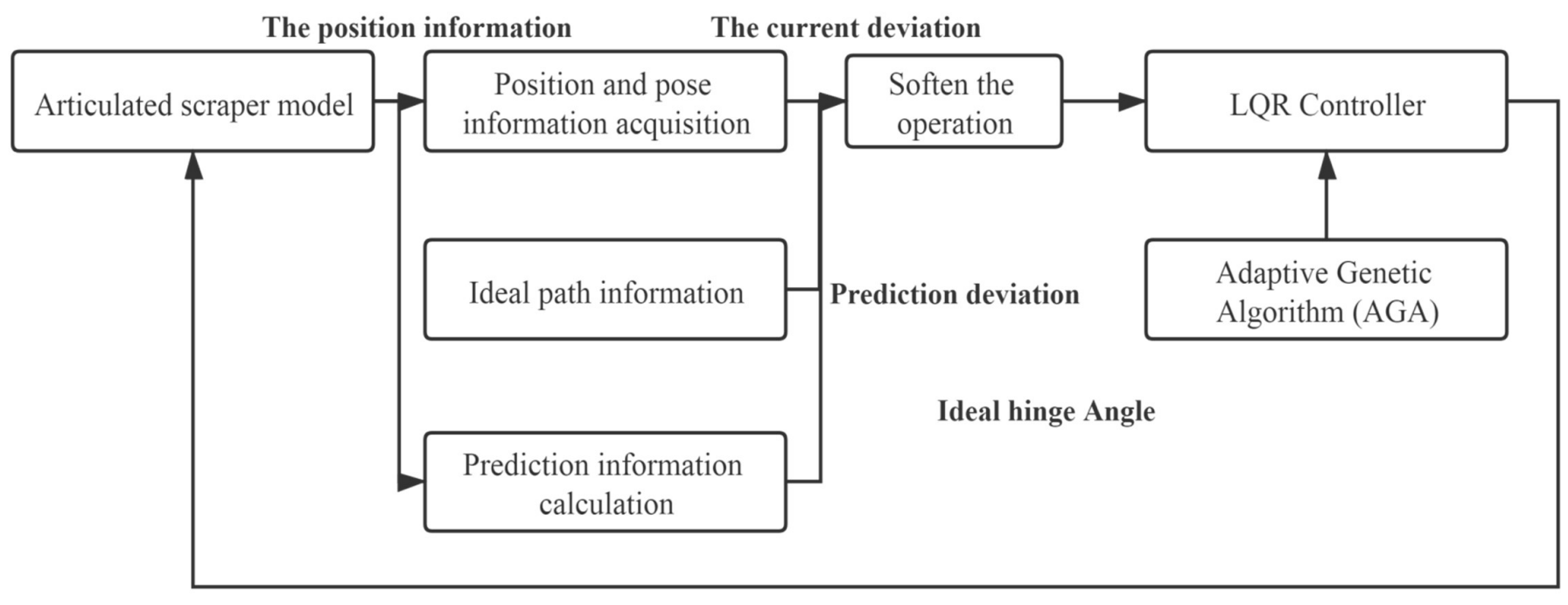

Ideal hinge Angle

Figure.7 LQR-AGA control system block diagram

\subsubsection{Simulation experiment of LQR-AGA control algorithm}

Used for the simulation of the path as shown in Figure 8, for wavy roadway, the halfway point of the cross section of the roadway in the attachment for the ideal of scraper run path which control target path, this path has continuous turning and other complex road conditions, so for the controller detection needs to have a strict conditions, to embody the scraper movement in actual operation[42]. In addition, in order to ensure the safe operation of the scraper, the maximum lateral deviation, namely the safe distance, should be set within $0.6 \mathrm{~m}$ [43].

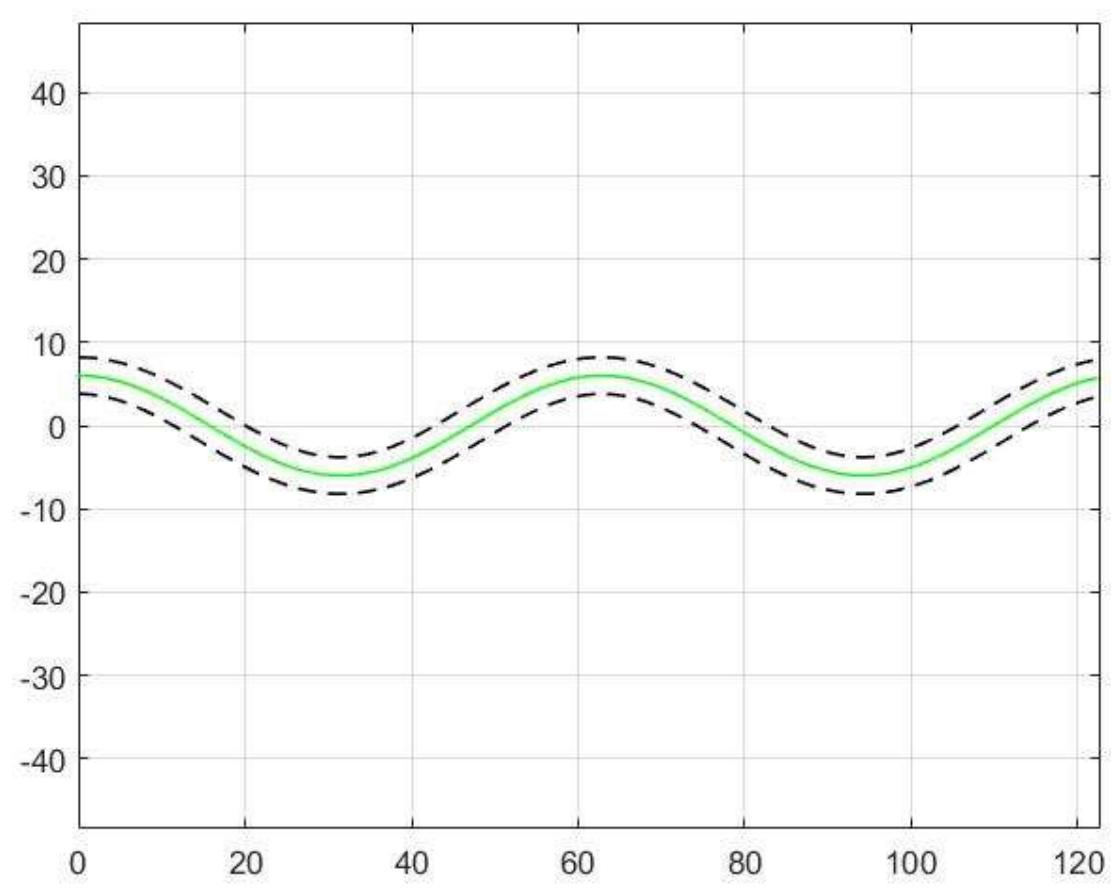

Figure.8 Simulation path of a downhole articulated scraper 
For example, it is assumed that the population size $\mathrm{N}$ is set to 30 and the number of iterations $\mathrm{G}$ can be set to 50 generations. The probability of crossover is about 0.2 , which can ensure that the population has strong adaptability, and can have a better global optimization ability and algorithm iteration speed. The adaptive variation constant 0.2 can ensure that the population has a relatively good global optimization ability, and avoid falling into local optimization in the iteration. The variation action constant $b=3$ guarantees the global capability at the initial stage of variation and ensures that the local optimum will not fall into at the end of variation. The survival range of the population $[0,50]$ ensures the positive nature of the control matrix and the stability of the whole control system[44].

In summary, all parameters of LQR-AGA are set as shown in Table 2 below.

Table 2. AGA parameter configuration

\begin{tabular}{cc}
\hline Parameter Name & $\begin{array}{c}\text { Numerical } \\
\text { value }\end{array}$ \\
\hline Population size: $\mathrm{N}$ & 30 \\
Iterations: $\mathrm{G}$ & 50 \\
Cross-crossing \\
probability \\
Adaptive variation \\
constant \\
Variiation \\
constconstant $\mathrm{b}$ \\
Population survival \\
range
\end{tabular}

The initial test coordinate of the articulated scraper is set as $[0.00,6.50]$, the initial test heading Angle is set as $0 \pi$, the hinged steering Angle is returned to zero, and the driving speed is constant $3.5 \mathrm{~m} / \mathrm{s}$. AGA algorithm is used to optimize the weighted matrix in the path tracking controller of the articulated scraper. After repeated experiments, we found that in the first 20 iterations of AGA algorithm, the population has already had strong spatial distribution and global search ability. The spatial distribution and variation are wide. In the last 20 generations, the population shows strong convergence and quickly converges to the optimal solution of living space. The adaptations of whole populations to humans and other creatures in nature are the same as the adaptations of humans to populations[45]. Table 3 shows the results of parameter optimization. The results show the historical optimal adaptability in the iteration time environment of other parameters. In the 50 iterations, the fitness of AGA algorithm showed monotonically increasing, indicating that both individuals and population were evolving towards the position of the optimal solution, and the fitness of the population remained stable at the end of the iteration, indicating that the entire population had converged to the optimal solution[46].

Table 3. AGA algorithm searching for optimal results

\begin{tabular}{llll}
\hline Algorithm & Weighted matrix $Q$ & Linear feedback matrix K & Suitability
\end{tabular}




\begin{tabular}{lccccccc} 
& $q_{1}$ & $q_{2}$ & $q_{3}$ & $k_{1}$ & $k_{2}$ & $k_{3}$ & \\
\hline AGA & 1.4685 & 33.5161 & 33.8515 & 1.0605 & 6.3752 & 6.5008 & 18220 \\
\hline
\end{tabular}

(A) Number of iterations 1

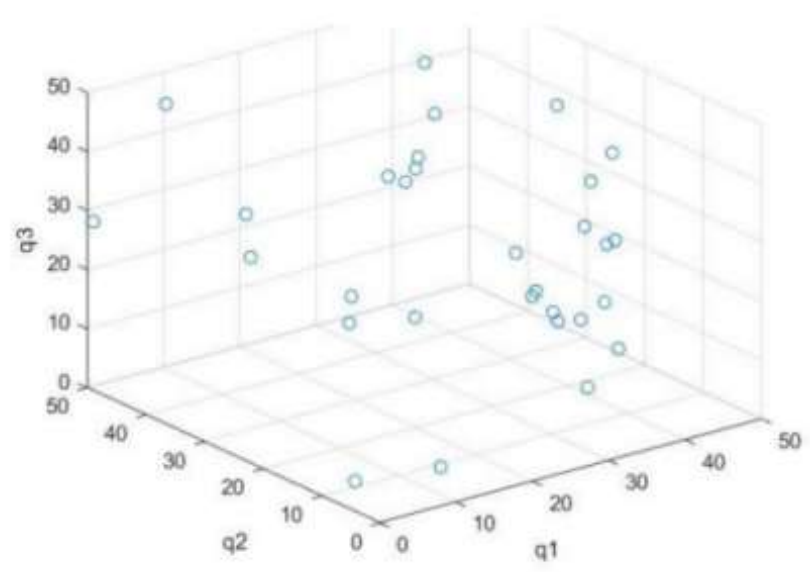

(C) Number of iterations 49

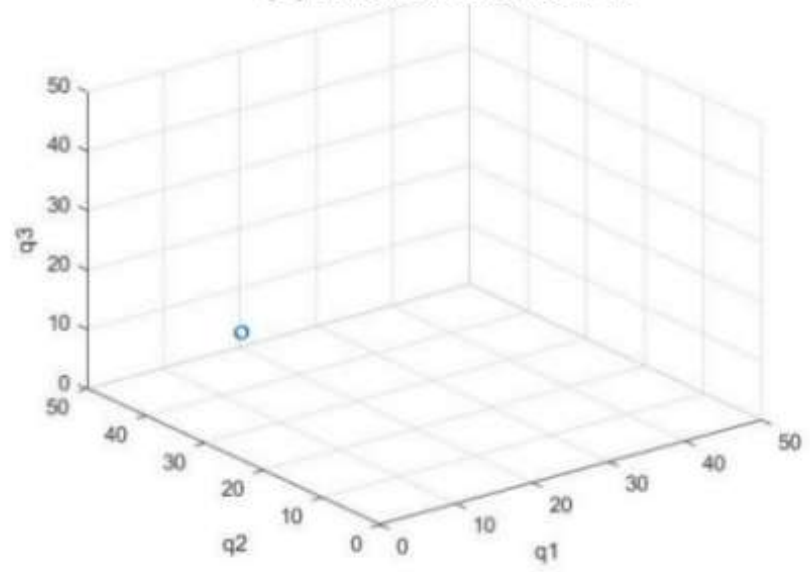

(B) Number of iterations 19

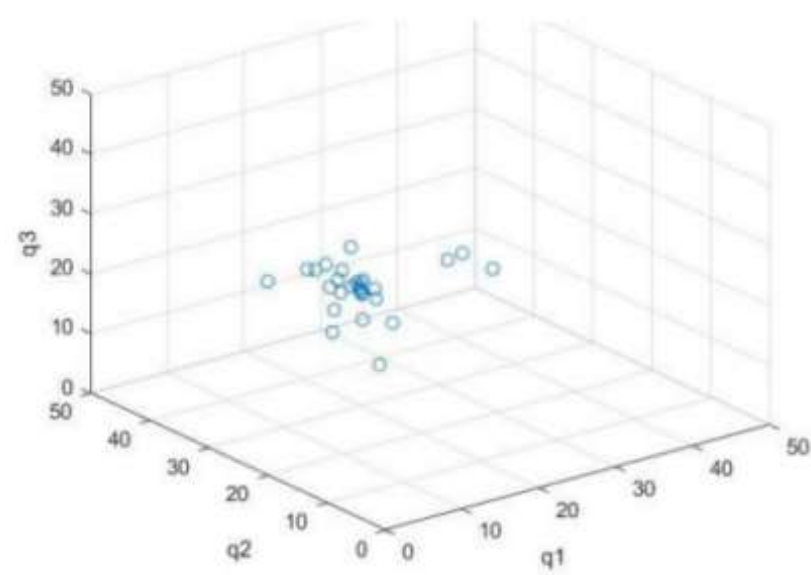

(D) Average fitness per generation

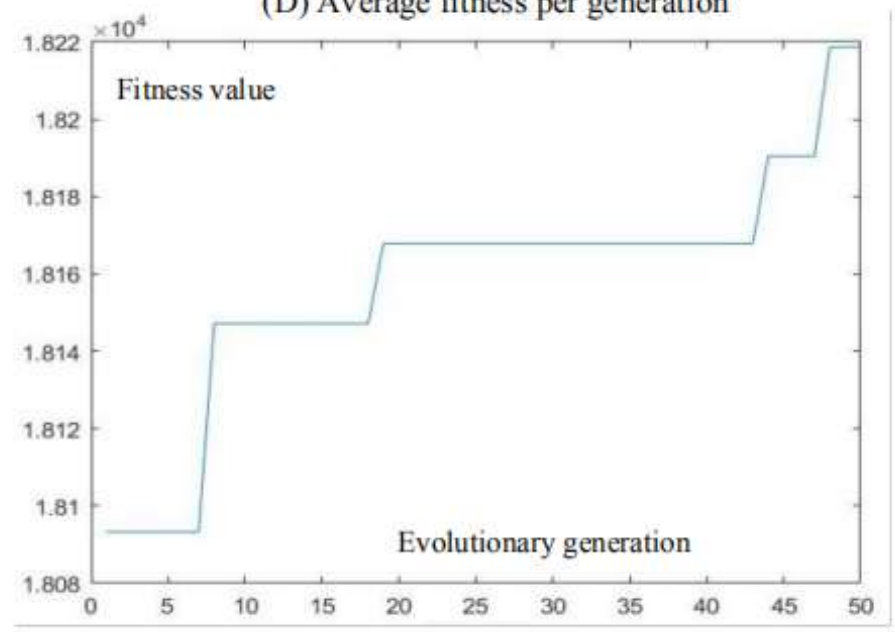

Figure.9 A,B,C: Population distribution of the 1st, 19th and 49th generations; D: Average fitness per generation. 


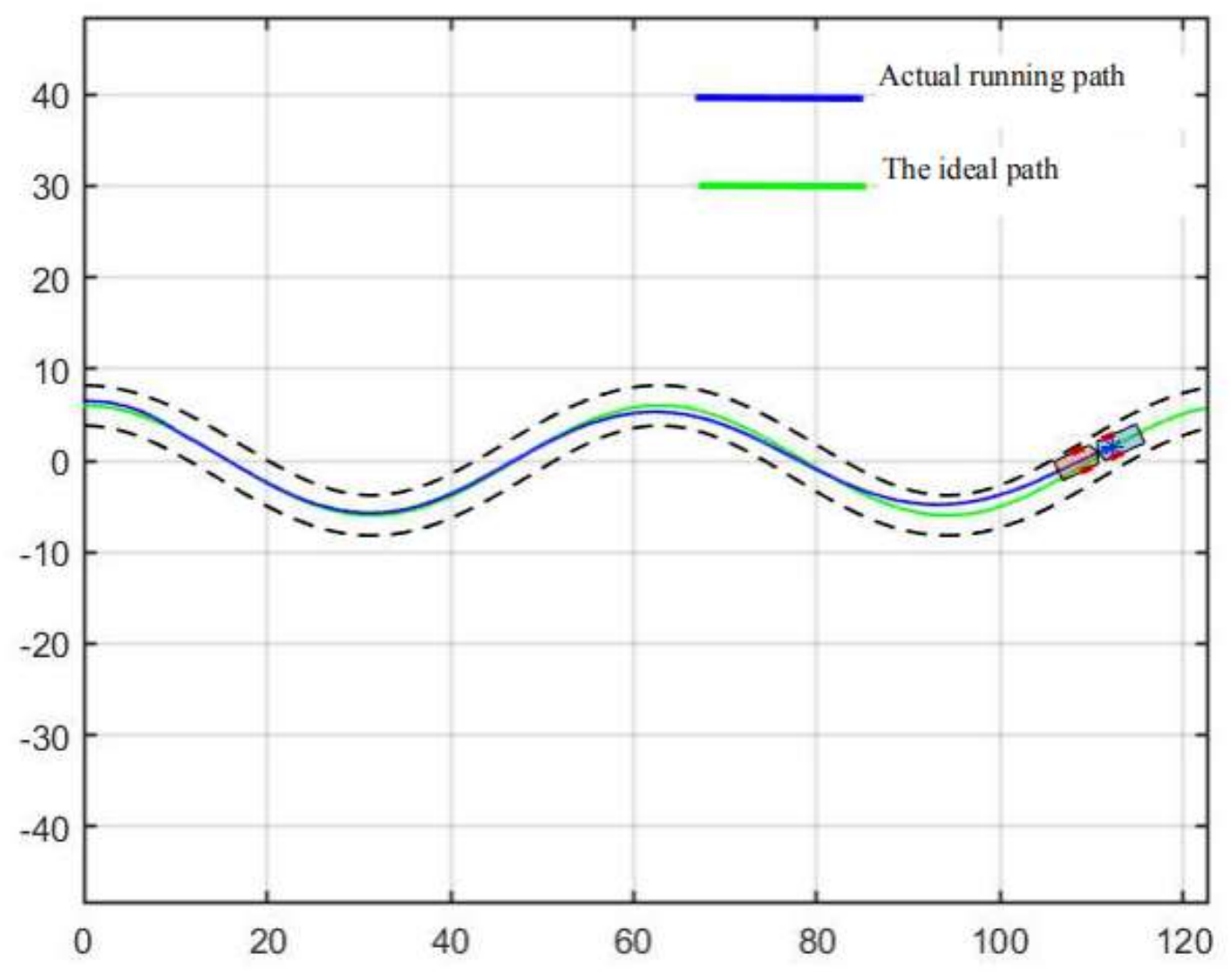

Figure. 10 Simulation result. 

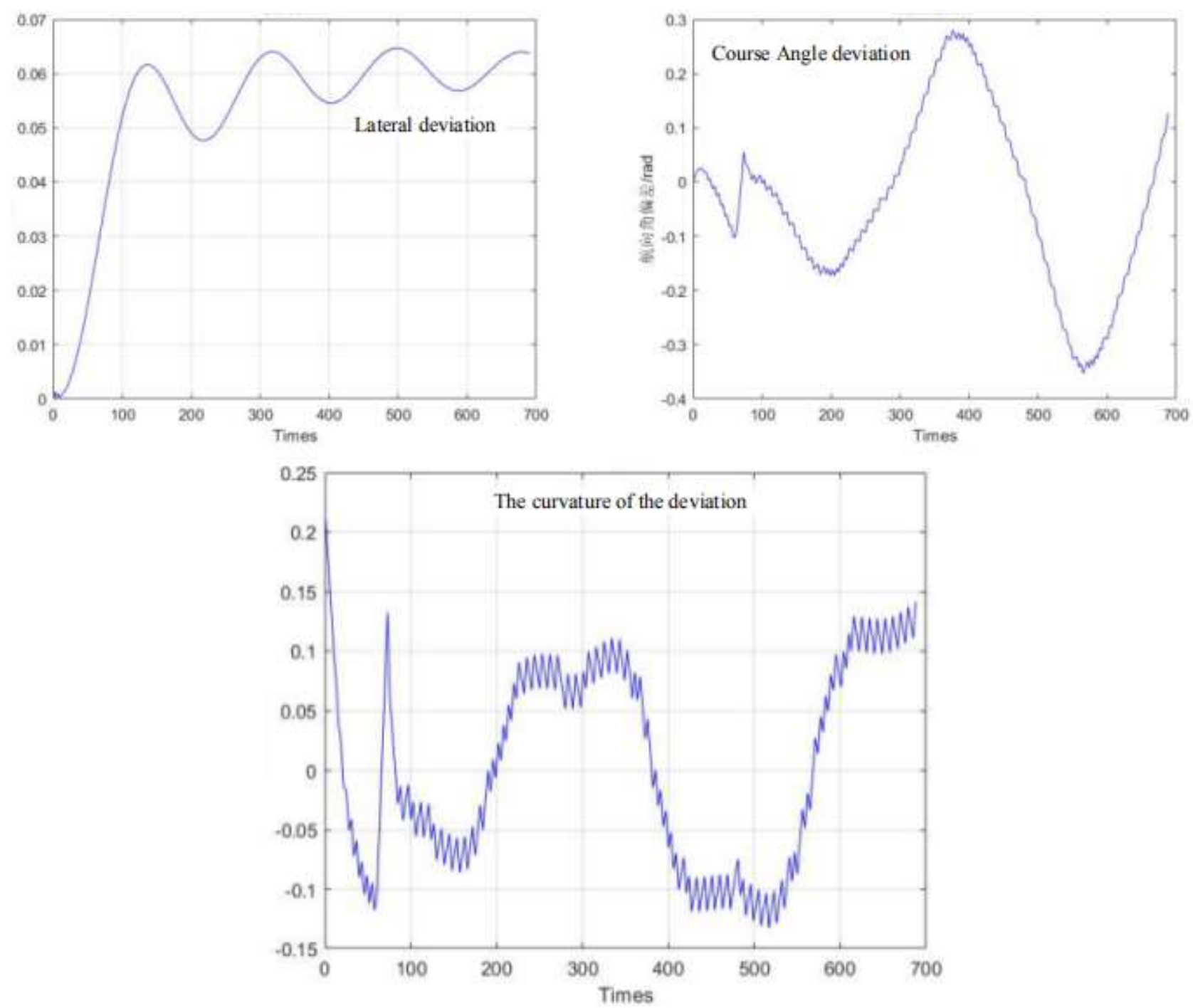

Figure. 11 Deviation range.

As can be seen from Fig.9 to Fig.11, the population has a strong global optimization ability at the beginning, and the convergence rate is fast at the later stage of iteration, and the average fitness of the population is high at the end of iteration. From the perspective of the simulation environment, the lateral error of the LHD on the simulated path is less than $0.1 \mathrm{~m}$, so it can be seen that the weighted matrix $Q$ optimized by the AGA algorithm makes the actual route of the articulated LHD basically coincide with the ideal planning path[47].

\subsection{Optimization of QPSO algorithm}

\subsubsection{Disadvantages of simple PSO algorithm}

The model of the simple PSO algorithm is the Boid (bird-oid) model of birds' predation behavior, which simulates the predation characteristics of the gregarious creatures. Because of its low requirement for the objective function, simple programming and easy programming, this model algorithm plays an important role in data scheduling optimization processing, function optimization analysis, intelligent training and neural network and other emerging disciplines. However, BOID model also has obvious disadvantages, such as serious precocity problem of population, strong randomness of optimization results, and the global optimal advantage can only be found when the number of iterations 
approaches infinity. These reasons will result in that the PSO algorithm of the BOID model cannot satisfy the parameter optimization function of the LQR controller, because the objective function of the LQR is a complex multi-peak function, and the randomness of solving it by the BOID model is too large, and it cannot guarantee that the optimization results can meet the path tracking requirements of the down-hole articulated scraper. Therefore, it is necessary to improve the simple PSO algorithm to achieve the optimization ability of LQR objective function[48].

\subsubsection{Quantum Behavior PSO Algorithm (QPSO)}

This particle swarm optimization algorithm strengthens the global optimization ability of each individual in the population. Combined with the linear decreasing weight strategy, the inertia of the individual group can be reduced at the end of the iteration to accelerate its convergence rate, so as to accelerate the group searching efficiency. The optimized particle swarm speed iterative algorithm is shown in Equation (30) :

$$
\left\{\begin{array}{c}
V_{i}=w V_{i}+a_{1} \operatorname{rand}()\left(\text { Pbest }-X_{i}\right)+a_{2} \operatorname{rand}()\left(\text { Gbest }-X_{i}\right) \\
X_{i}=X_{i}+V_{i} \\
W=\left(W_{\text {ini }}-W_{\text {end }}\right) \times \frac{G_{k}-g}{G_{k}}+W_{\text {end }}
\end{array}\right.
$$

Note: $V_{i}$ represents the velocity of the $i$ th particle; $X_{i}$ represents the location of the $i$ th particle; Pbest represents the historical optimal position of the particle; Gbest represents the historical optimal location of the population.

In view of the particle swarm optimization algorithm (PSO) in the late iteration of high dimension, it is easy to fall into local optimum and other problems. Therefore, the concept of hand velocity factor needs to be introduced to increase the velocity of particles at the end of iteration. Its formula is shown in Equation (31):

$$
V_{i}=K\left[V_{i}+a_{1} \operatorname{rand}(\quad) \times\left(P_{i}-V_{i}\right)+a_{2} \operatorname{Rand}() \times\left(P_{g}-X_{i}\right)\right.
$$

In the early stage of algorithm iteration, because the particle distribution is relatively scattered and the particle has a large inertia weight at this time, it will explore the space globally according to its initial test velocity, and the particle at this time has a strong global exploration ability. Therefore, the need for $\mathrm{K}$ worth configuration should be large initially. In the late iteration of the algorithm, the population needs to have strong convergence characteristics, so as to speed up the operation speed of the algorithm, and the population needs to change in a small spatial range. To sum up, K value should show a monotonically decreasing characteristic with the increase of the number of iterations, so we can set the function of $\mathrm{K}$ value changing with the number of iterations as shown in Equation (32).

$$
K=\frac{\cos \left(\left(\frac{T}{\max \_G e n}\right) T\right)+2.5}{4}
$$

Note: T represents Number of current iterations; Max_Gen represents Maximum number of iterations.

Quantum-behaved particle swarm optimization (QPSO the simple PSO algorithm adjusted the position update strategy, its cancelled this property, instead, the probability distribution function, which means that the particles according to the probability distribution, rather than according to the velocity distribution, so the space attribute need each particle in a population by each observation to determine. The formula for calculating the average value of the historical optimal fitness of a single particle is as follows:

$$
m=\frac{1}{N} \sum_{i}^{N} \text { pest }_{i}
$$


Note: $N$ is the Sum of the number of particles; Pest $t_{i}$ represents the optimal fitness of a single particle in the $i$ th iteration.

The position update of particles is based on the probability distribution function, as shown in Equation (34) :

$$
\left\{\begin{array}{c}
P_{i}=\xi \times \text { pest }_{i}+(1-\xi) \times \text { gest }_{i} \\
X_{i}=P_{i} \pm a\left|m-X_{i}\right| \times \ln \left(\frac{1}{b}\right)
\end{array}\right.
$$

Note: $\xi$ represents the probability function obeys the uniform distribution on $(0,1)$; $\pm \alpha$ represents the expansion coefficient, the probability of positive is $50 \%$, and the probability of negative is $50 \%$.

Particle swarm optimization analysis algorithms directly using particle group each particle in the individual information is used to group comprehensive analysis and information sharing of the information to directly promote the coordinated motion of particle groups, making it directly in a population problem solving process in space will naturally produce directly from disorderly to orderly way of the evolution process of evolution, Thus, we can directly obtain the optimization and understanding of a group problem, whose basic flow A and block diagram B of the LQR-QPSO system are shown in Figure 12 and Figure 13.

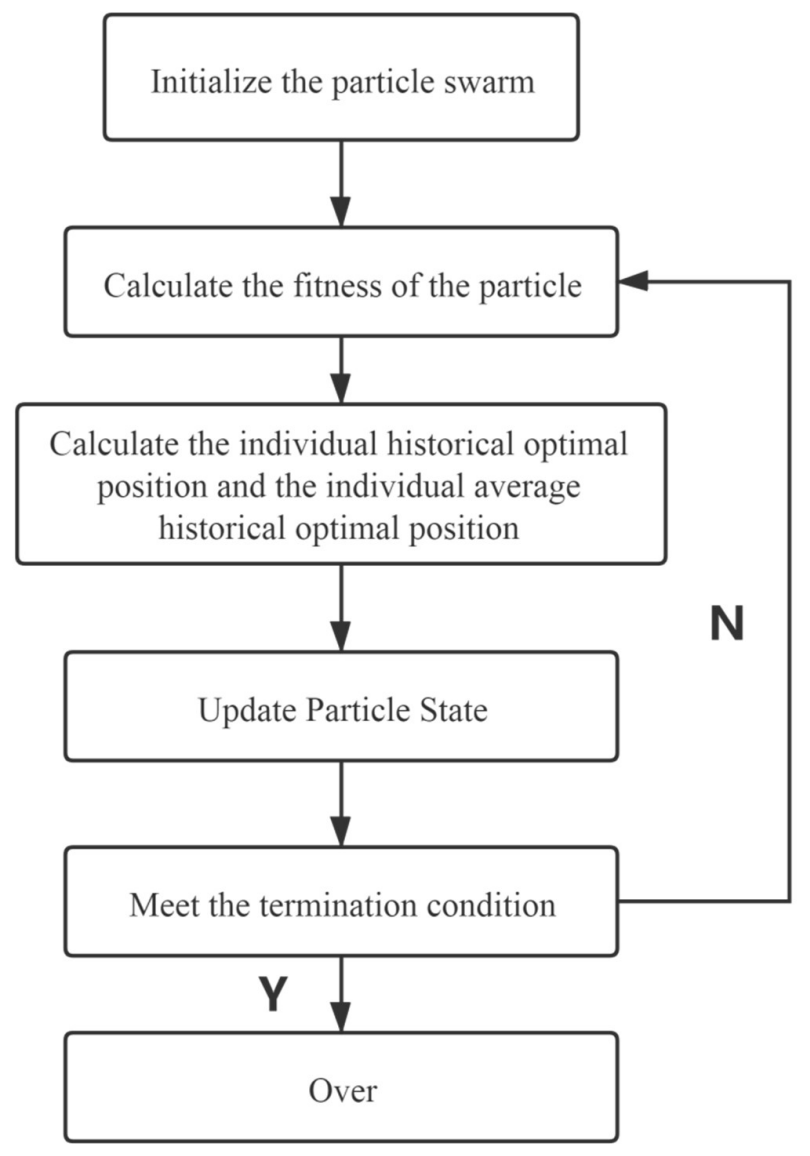

Figure. 12 Particle Group algorithm algorithm Flow. 


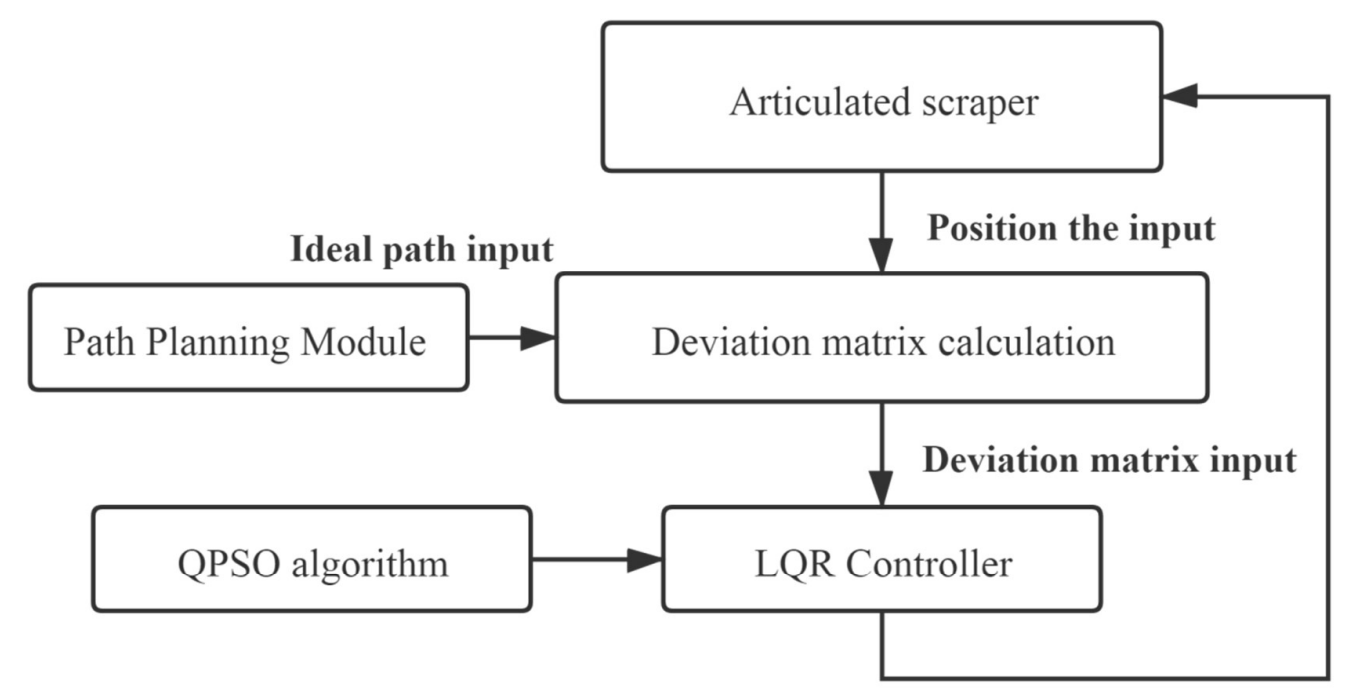

\section{Angular velocity of turning}

Figure. 13 Particle Group algorithm algorithm Flow.

4.2.3. LQR-QPSO control algorithm simulation experiment

The simulation environment is shown in Figure 8; See Table 1 for body parameters of the downhole articulated scraper; QPSO parameter configuration is shown in Table 4. The maximum lateral deviation is required to be less than $0.6 \mathrm{~m}$ [49].

Table 4. QPSO configuration of quantum behavior particle group algorithm parameters

\begin{tabular}{|c|c|}
\hline Parameter Name & Numerical value \\
\hline Iterations: $\mathrm{G}$ & 80 \\
\hline Population size: $\mathrm{N}$ & 30 \\
\hline $\begin{array}{l}\text { Termination of the inertia } \\
\text { weight } \mathrm{w}_{\text {end }}\end{array}$ & 0.4 \\
\hline Initial inertia weight $w_{\text {ini }}$ & 0.9 \\
\hline Learning Factors $a_{1}$ & 1.5 \\
\hline Learning Factors $\mathrm{a}_{2}$ & 1.5 \\
\hline $\begin{array}{l}\text { Particle Taking Value } \\
\text { Limitq }_{\max }\end{array}$ & 400 \\
\hline Max imum speedv $v_{\max }$ & 1.0 \\
\hline $\begin{array}{l}\text { Initial shrinkage-factor of ex- } \\
\text { pansion } \alpha_{b}\end{array}$ & 1.0 \\
\hline
\end{tabular}


Termination shrinkage-Expansion ation factor $\alpha_{\mathrm{e}}$

The initial test coordinate of the articulated scraper is set as $[0.00,6.50]$, and the heading Angle of the initial test is set as zero Angle, namely $0^{\circ}$. In addition, the steering Angle of the front and rear car bodies of the articulated scraper is set to zero, which means that the body keeps moving forward, and the traveling speed is $3.5 \mathrm{~m} / \mathrm{s}$, so it is inconvenient to keep the speed constant. After repeated trial and simulation tests, it is found that in all experiments, the population convergence rate of QPSO algorithm is slow. In the first 30 generations, each particle varies greatly in the global scope, showing obvious divergence, but after 60 generations, the particle swarm gradually converges to the optimal value. And the controller parameters obtained in the 80 generation can make the errors in the operation process of the articulated scraper within a reasonable range[50].

Table 5. Optimization results of QPSO algorithm

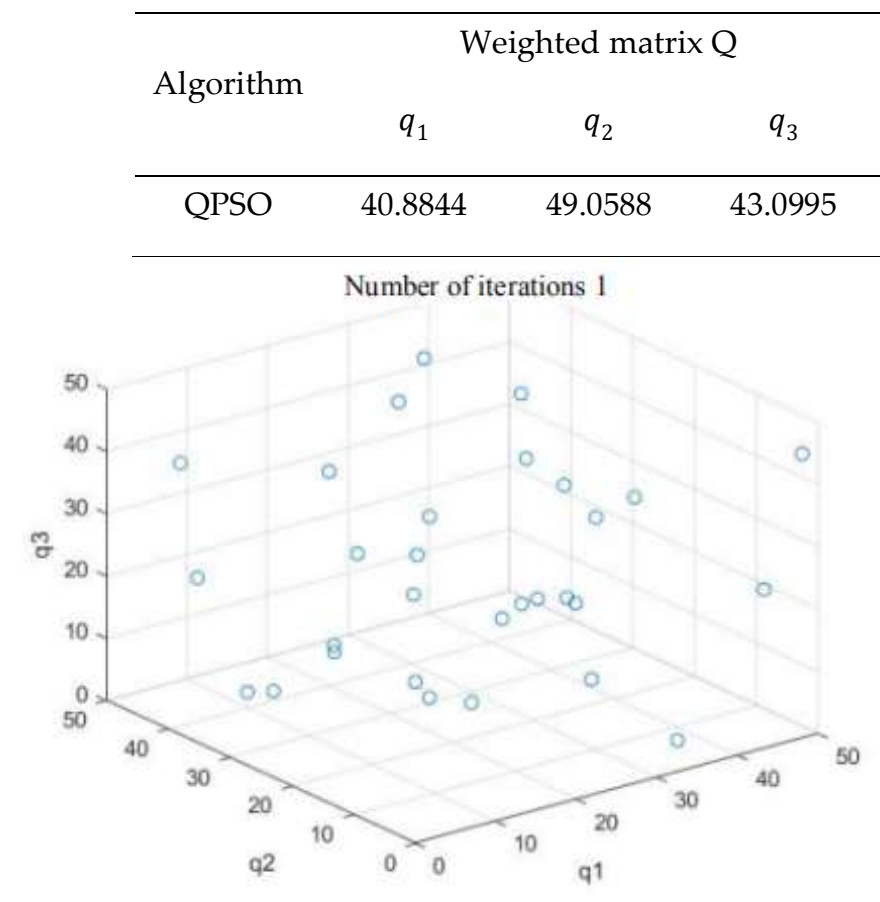

\begin{tabular}{cccc}
\multicolumn{3}{c}{ Linear feedback matrix $\mathrm{K}$} & Suitability \\
$k_{1}$ & $k_{2}$ & $k_{3}$ & \\
\hline 5.2700 & 10.6900 & 6.7451 & 17330
\end{tabular}
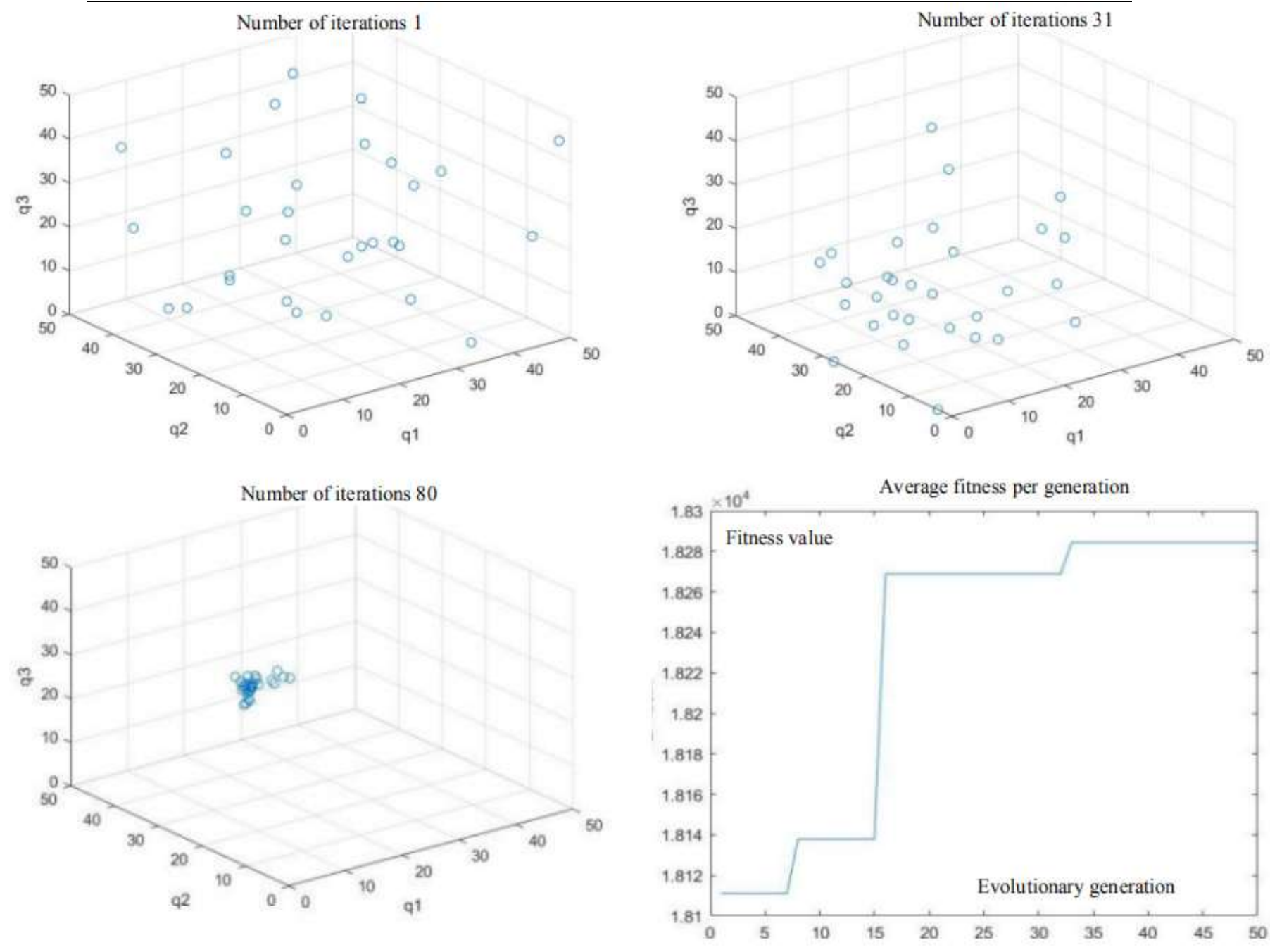
Figure. 14 Population distribution of the 1st, 31th and 80th generations; and Average fitness per generation.

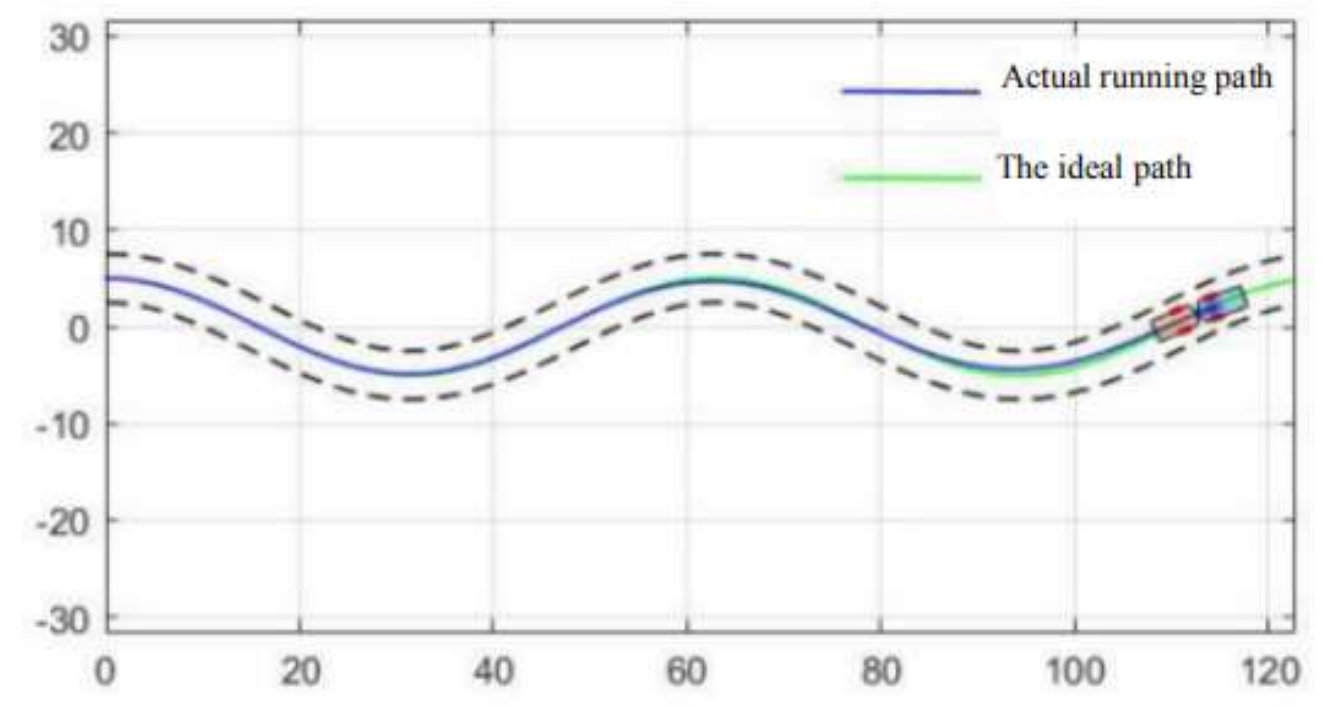

Figure. 15 Simulation results.
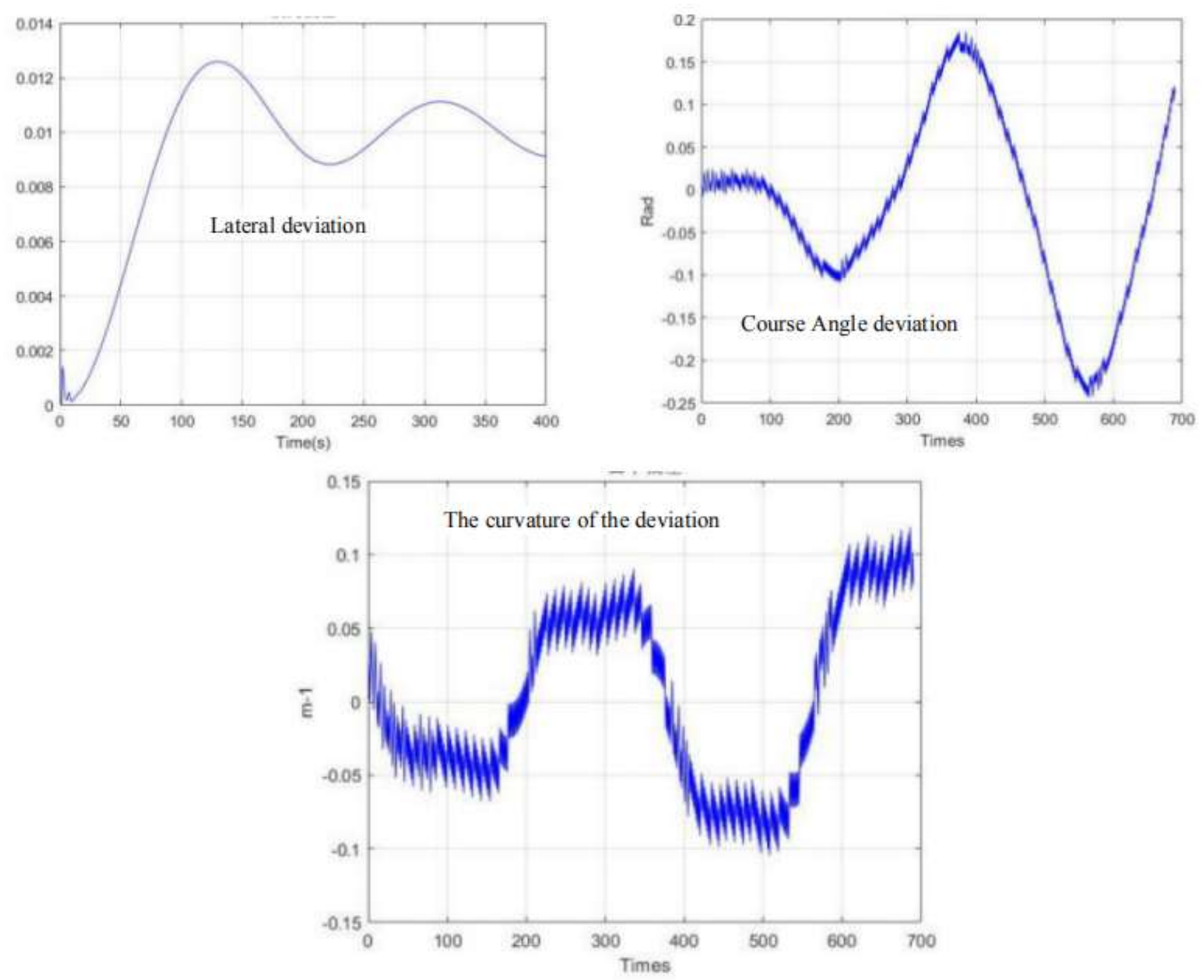
Figure. 16 Deviation range.

\subsection{ACA optimization of ant colony algorithm}

4.3.1. Ant Colony Algorithm - LQR Controller (LQR-ACA)

An LQR-ACA path tracking controller can be established as shown in Figure 17[51].

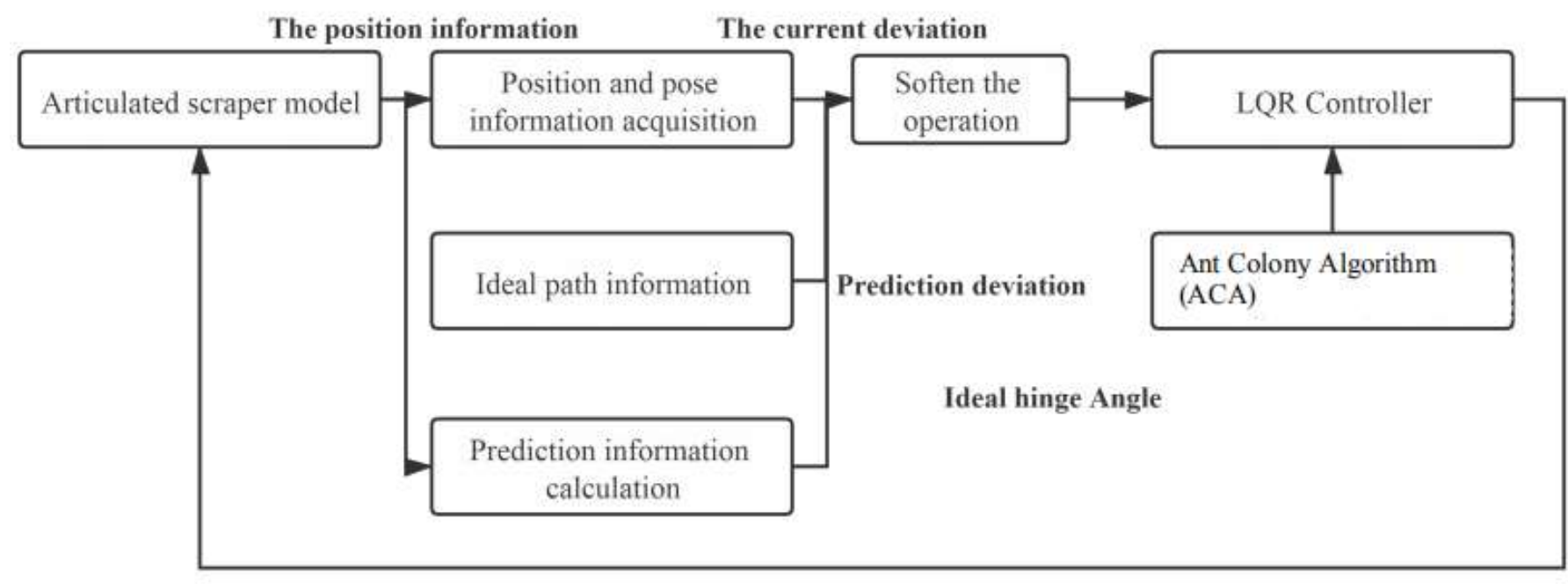

\section{Ideal hinge Angle}

Figure. 17 LQR-ACA control system block diagram.

4.3.2. LQR-ACA control algorithm simulation experiment

The validity and reliability of the LQR-ACA path traceability controller were tested and verified by MATLAB simulation[52]. In order to make this ant group have better search ability and code iteration speed, Ant number is set as 30, and search times G is set as 50 generations. Hormone play factor is set to 0.4 and the search range is set to $[0,50]$ [53].

Table 6. ACA parameter configuration

\begin{tabular}{cc}
\hline Parameter Name & Numerical value \\
\hline Ant Number: ant & 30 \\
Search times: $G$ & 100 \\
hormone play factor $w_{i n i}$ & 0.4 \\
Transfer probability $P_{0}$ & 0.2 \\
\hline
\end{tabular}

The initial coordinate of the articulated scraper is set as [0.00,6.50], the heading Angle of the initial test is set as $0 \pi$, the hinged steering Angle is returned to zero, and the driving speed is constant $3.5 \mathrm{~m} / \mathrm{s}$. Ant colony algorithm is used to configure the parameters of the $\mathrm{LQR}$ controller of the articulated scraper. After tested repeatedly found that ant colony algorithm of ant colony in 100 iterations will converge to different extreme value point, and the position of most of the ants in the number of iterations is more than 20 since he 
no longer changes generation, the fitness function of the LQR controller is a more extreme value point function, there were many in the solution space fitness equal points[54].

Table 7. ACA algorithm optimization results

\begin{tabular}{cccccccc}
\hline & \multicolumn{3}{c}{ Weighted matrix Q } & \multicolumn{2}{c}{ Linear feedback matrix K } & & Suitability \\
& $q_{1}$ & $q_{2}$ & $q_{3}$ & $k_{1}$ & $k_{2}$ & $k_{3}$ & \\
\hline Algorithm C A & 0.8419 & 7.0752 & 40.6476 & 0.8288 & 4.1523 & 6.5550 & 15333 \\
\hline
\end{tabular}
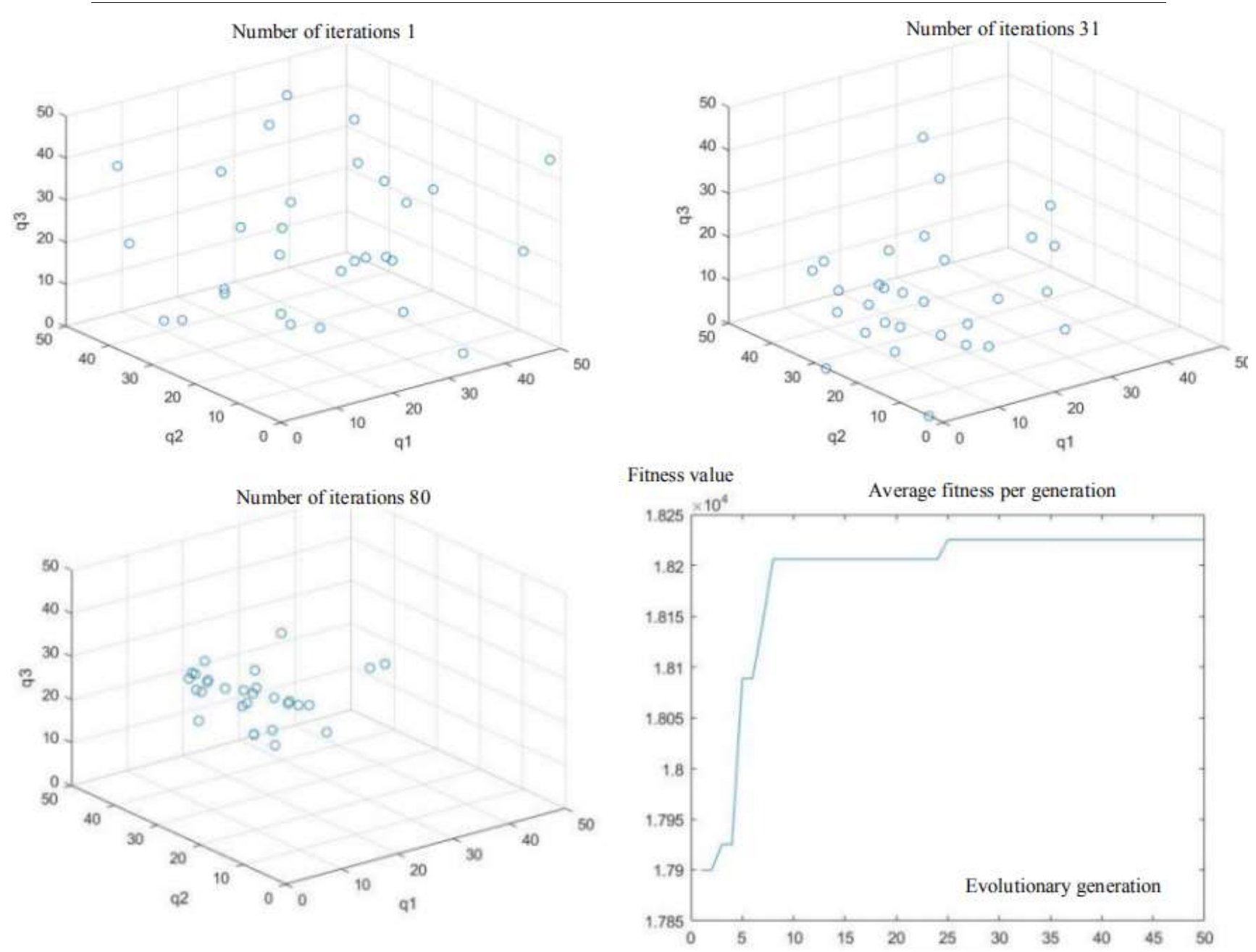

Figure. 18 Population distribution of the 1st, 31th and 80th generations; and Average fitness per generation.

The parameter optimization results are brought into the simulation environment of the articulated scraper to complete the path tracking simulation, and the results as shown in Figure 19 can be obtained. The weighted matrix Q obtained by the ACA algorithm makes the actual route of the articulated scraper coincide with the ideal planning path basically[55]. 


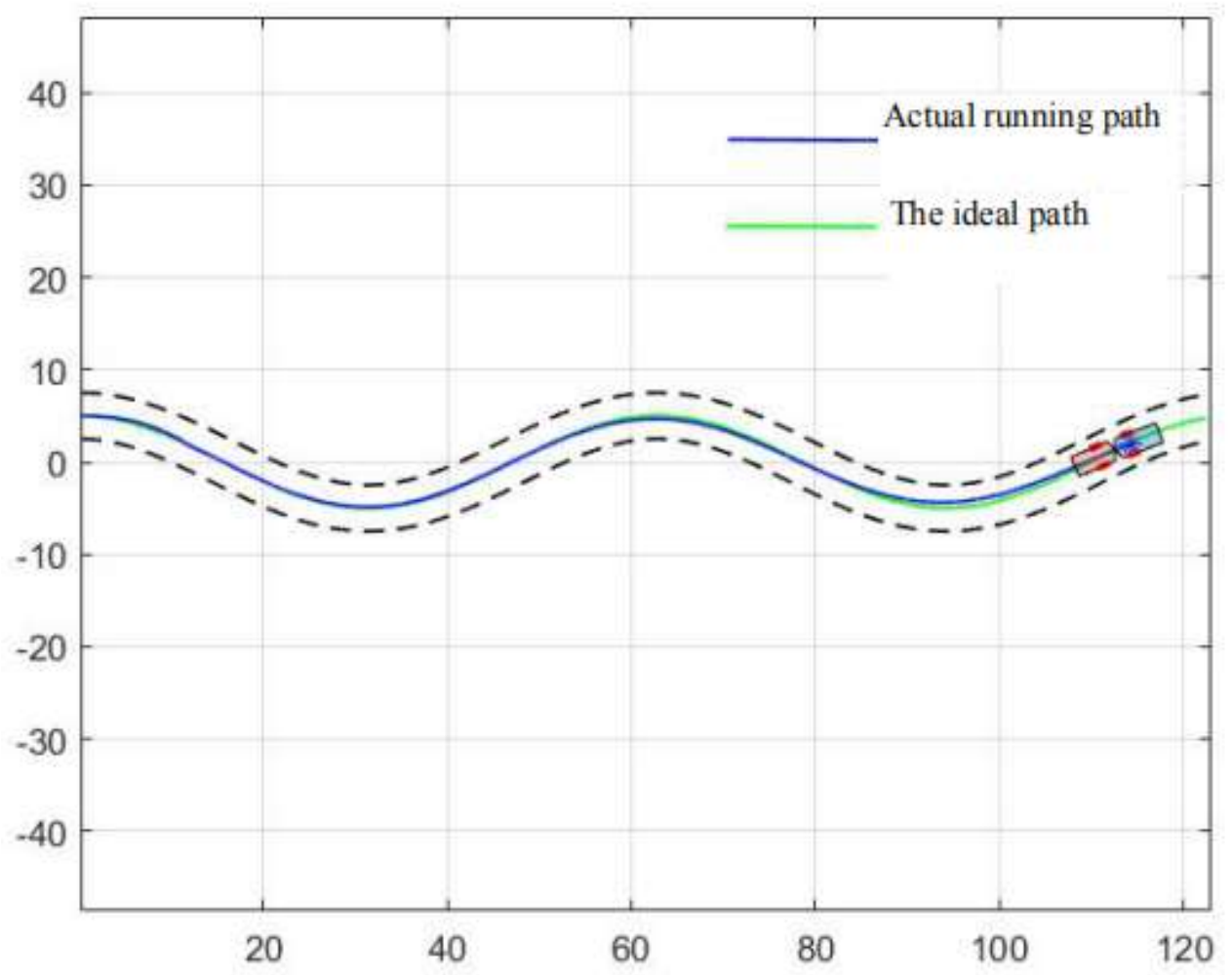

Figure. 19 Simulation results. 


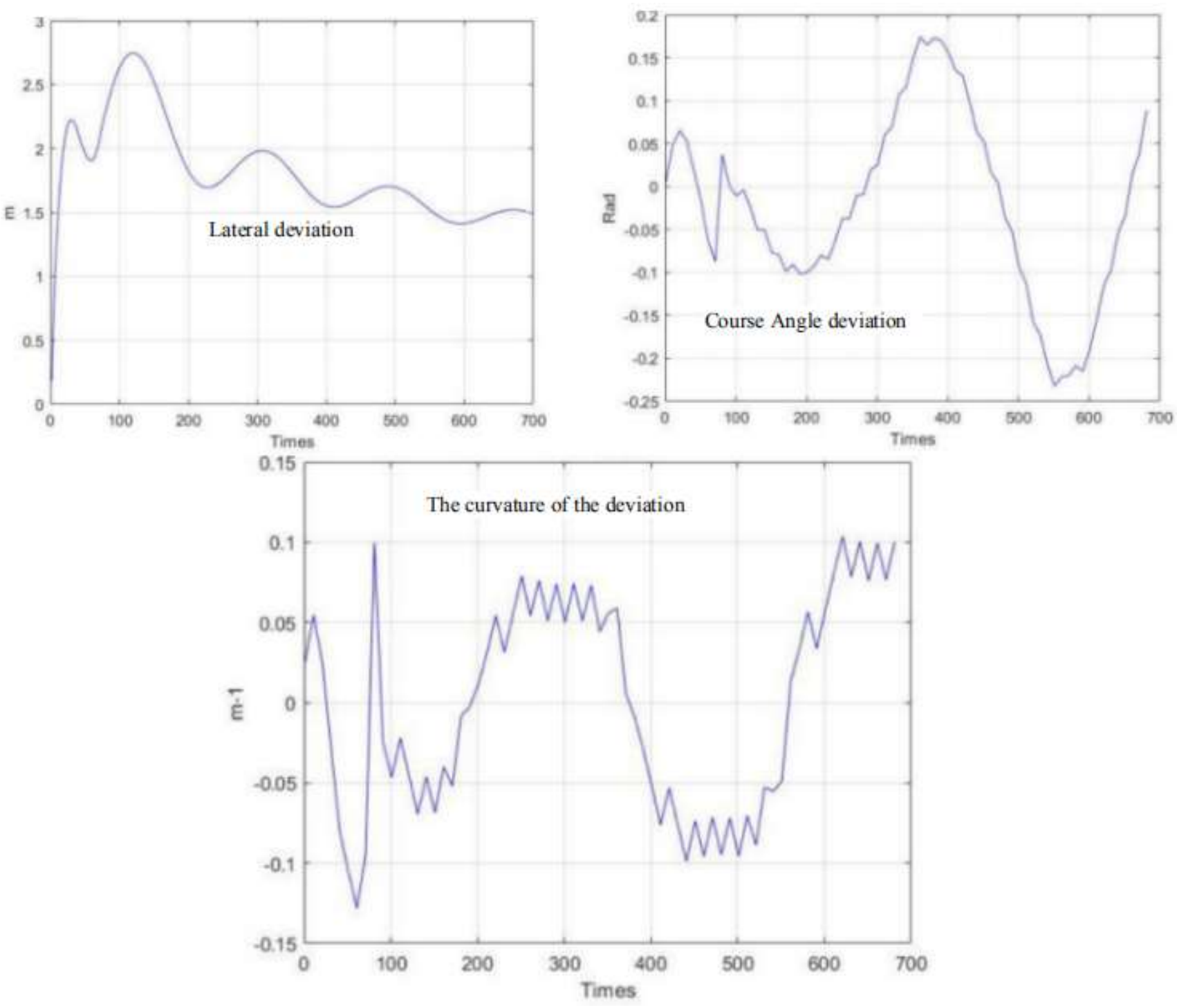

Figure. 20 Deviation range.

\section{Comparison of intelligent cluster algorithm optimization LQR}

\subsection{Comparison of algorithm parameter configuration}

The population size of AGA, QPSO and ACA was 30. Both AGA and QPSO populations converge to a certain extreme point, but AGA population converges faster than QPSO, while ACA population converges to multiple extreme points, and the calculation time is longer.

Table 8. Parameter configuration comparison

\begin{tabular}{cccc}
\hline Algorithm Name & Population size & $\begin{array}{c}\text { Number of } \\
\text { convergence } \\
\text { iterations }\end{array}$ & Operation time \\
\hline Adaptive Genetic algorithm AGA & 30 & 50 & $15 \mathrm{~min}$
\end{tabular}


Quantum behavior particle swarm algorithm QPSO

Ant colony algorithm ACA
30

30
80

$25 \mathrm{~min}$

\subsection{Comparison of algorithm results}

The parameter configuration of $Q$ matrix obtained by the three intelligent clustering algorithms is very different, but they all have high fitness. AGA had the highest fitness, while ACA had the lowest fitness.

Table 9. Comparison of algorithm optimization results

\begin{tabular}{cccccccc}
\hline & \multicolumn{3}{c}{ Weighted matrix Q } & \multicolumn{3}{c}{ Linear feedback matrix K } & \multicolumn{2}{c}{ Suitability } \\
& $q_{1}$ & $q_{2}$ & $q_{3}$ & $k_{1}$ & $k_{2}$ & $k_{3}$ & \\
\hline AGA & 1.4685 & 33.5161 & 33.8515 & 1.0605 & 6.3752 & 6.5008 & 18220 \\
\hline QPSO & 40.8844 & 49.0588 & 43.0995 & 5.2700 & 10.6900 & 6.7451 & 17330 \\
\hline A C A & 0.8419 & 7.0752 & 40.6476 & 0.8288 & 4.1523 & 6.5550 & 15333 \\
\hline
\end{tabular}

\subsection{Comparison of simulation results}

In the simulation environment, the ideal path of the articulated scraper is a circular trajectory with $(0,0)$ as the center of the circle and a radius of 5 , as shown in Figure 21 . It can be seen from Figure 22 that the optimization results of the three clustering algorithms can all meet the error requirements. In the simulation, the initial error of the optimization results obtained by the AGA algorithm and the ACA algorithm is large. After about a quarter of a semicirculate trajectory, the optimization controllers obtained by the AGA and ACA intelligent clustering algorithms can reduce the deviation degree between the LHD and the ideal path in the subsequent operation through the control effect. The optimization results obtained by the QPSO algorithm are always less deviated from the ideal path. 


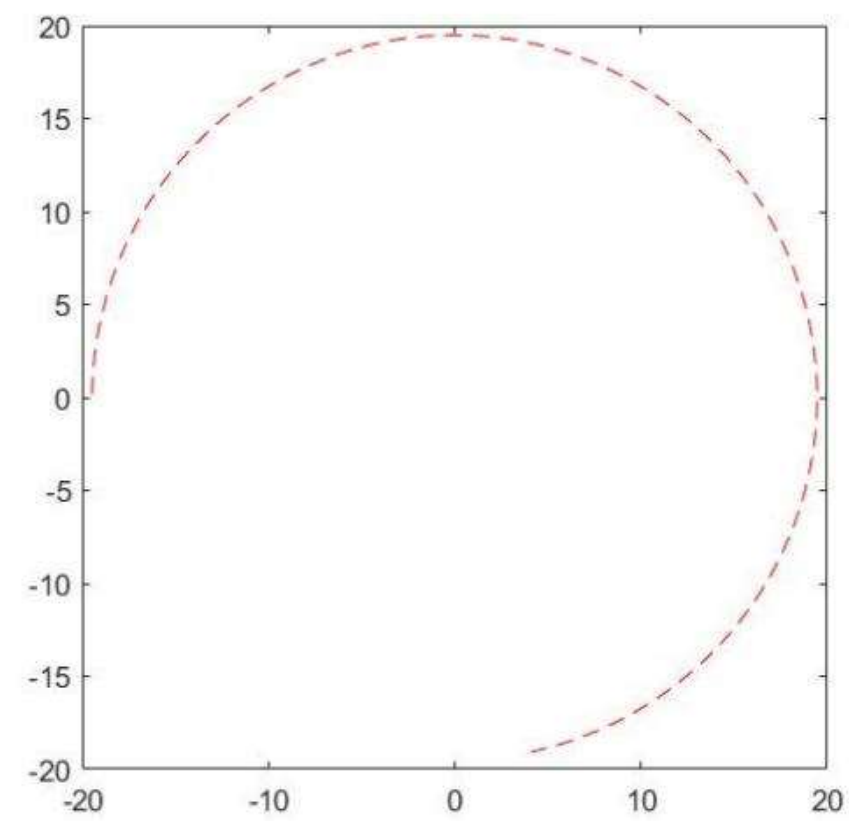

Figure. 21 Contrast experimental simulation paths. 


\section{AGA simulation results}

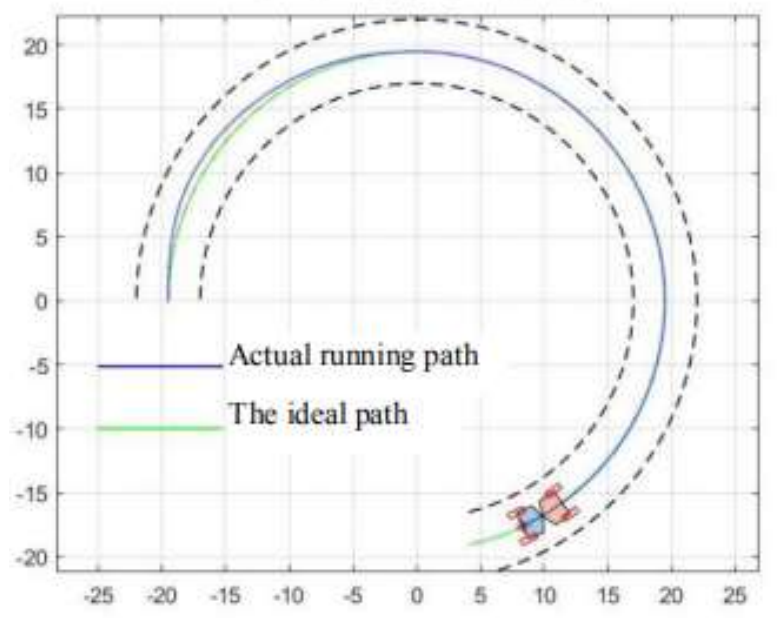

\section{QPSO simulation results}

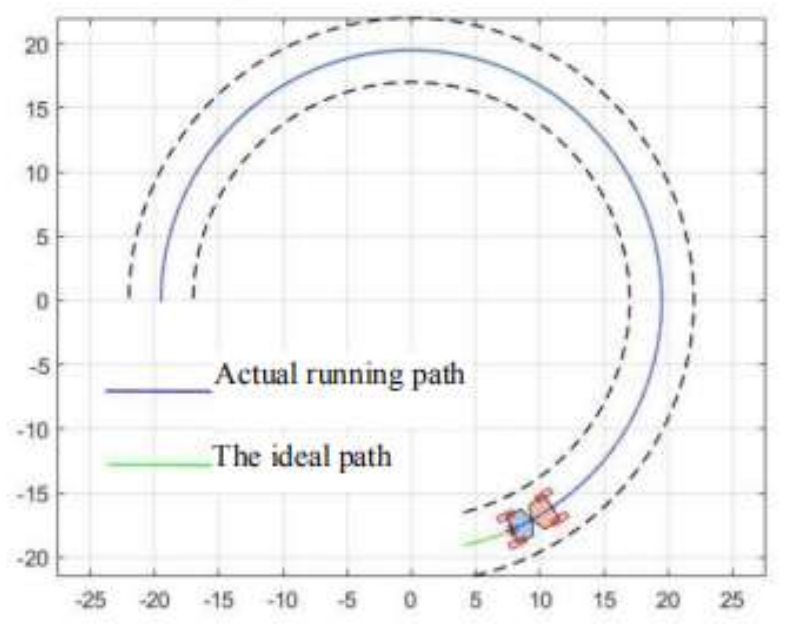

ACA simulation results

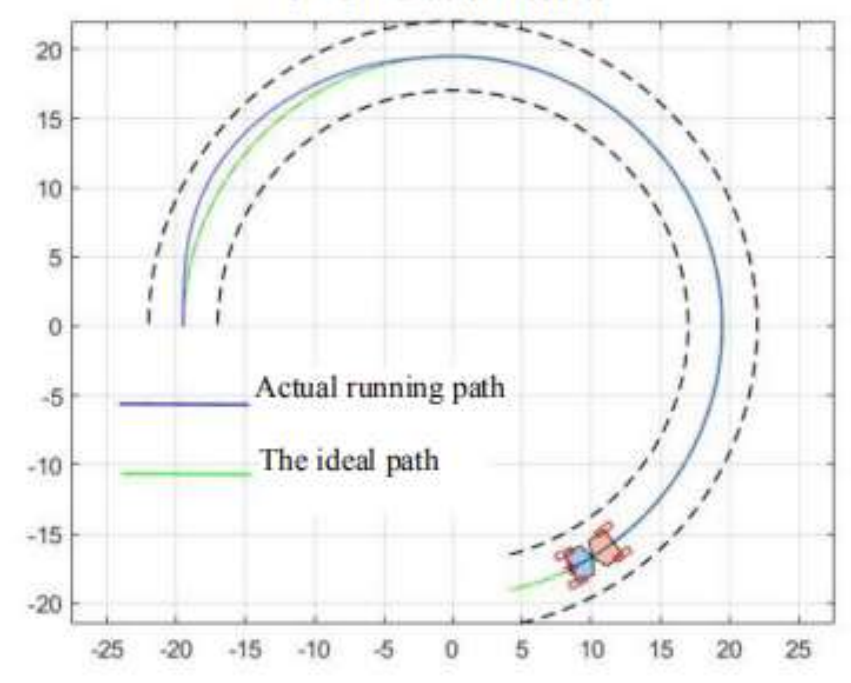

Figure. 22 Comparison simulation results.

\section{Conclusion}

AGA, QPSO and ACA intelligent clustering algorithms can all find the optimal results for LQR parameter configuration problem, but these three algorithms have their own advantages and disadvantages. For example, the AGA algorithm has fast operation speed and fast group convergence, but the optimization result is poor compared with the QPSO algorithm. QPSO algorithm is slow in operation speed and group convergence speed, but it can find the optimal solution. ACA algorithm is slow in calculation speed and group convergence speed, but it can converge to multiple extreme points, and there is a large space for optimization.

The biggest characteristic of intelligent clustering algorithm is the problem of data prematurity. Secondly, in terms of LQR parameter configuration, the fitness function is the main reason for the slow operation speed of intelligent clustering algorithm. In order to solve the precocity problem, the development direction of this kind of algorithm is to improve the intelligent cluster algorithm, while the problem of computing speed is to simplify and redefine the fitness function of LQR to reduce the computing time. 
This paper studies the path tracking control of intelligent scraper unmanned driving technology, focusing on the linear quadratic optimal control LQR and the parameter optimization of intelligent clustering algorithm AGA, QPSO and ACA. The main work of this paper is summarized as follows:

(1) for articulated LHD path tracking control problems, this paper studied the kinematics model of the articulated LHD body, through the analysis of the kinematics modeling, it is concluded that the articulated LHD vehicle reference speed of anchor point, course angular velocity, turning angular velocity and the mathematical relationship between the scraper speed and steering angular velocity.

(2) for the selection of control scheme, based on the kinematics model of the articulated LHD lateral error identified the scraper, and heading Angle error, the error between the steering Angle and curvature of state space, according to the state space is put forward to steering Angle to control to control the amount of articulated LHD vehicle location of LQR controller, linear quadratic linear optimal control.

(3) Aiming at the problem that the parameters of LQR controller are difficult to select, this paper proposes the LQR controller scheme optimized by the intelligent cluster algorithm, and compares the advantages and disadvantages of different clustering algorithms, and puts forward a feasible implementation scheme for the path tracking control of the intelligent scraper.

Funding: This research received no external funding.

Acknowledgments: Special thanks to the editors for long-term guidance to authors.

Conflicts of Interest: The authors declare no conflict of interest.

Author Contributions: Y.Z.-Conceptualization/Resources. C.Z. - Writing review and editing/Visualization. Z.W. - Writing original draft/Validation. H.Y.-Writing review and editing/Project administration/Validation/Supervision. All authors have read and agreed to the published version of the manuscript.

\section{References}

[1] Li J, Zhan K. Intelligent mining technology for an underground metal mine based on unmanned equipment[J]. Engineering, 2018, 4(3): 381-391.

[2] Amer N H, Zamzuri H, Hudha K, et al. Modelling and control strategies in path tracking control for autonomous ground vehicles: a review of state of the art and challenges[J]. Journal of intelligent \& robotic systems, 2017, 86(2): 225254.

[3] Wang G, Xu Y, Ren H. Intelligent and ecological coal mining as well as clean utilization technology in China: Review and prospects[J]. International Journal of Mining Science and Technology, 2019, 29(2): 161-169.

[4] Paterson A D J, Joseph T G, Nychka J A, et al. Performance evaluation of ultra-class mining shovel track roller paths[J]. CIM Journal, 2020, 11(1): 9-19.

[5] Dutta T, Kim K H, Uchimiya M, et al. Global demand for rare earth resources and strategies for green mining[J]. Environmental Research, 2016, 150: 182-190.

[6] Shi J, Sun D, Qin D, et al. Planning the trajectory of an autonomous wheel loader and tracking its trajectory via adaptive model predictive control[J]. Robotics and Autonomous Systems, 2020, 131: 103570. 
[7] Fancher P, Winkler C. Directional performance issues in evaluation and design of articulated heavy vehicles[J]. Vehicle System Dynamics, 2007, 45(7-8): 607-647.

[8] Oreh S H T, Kazemi R, Azadi S. A new desired articulation angle for directional control of articulated vehicles[J]. Proceedings of the Institution of Mechanical Engineers, Part K: Journal of multi-body dynamics, 2012, 226(4): 298-314.

[9] Tian J, Wang S, Wu M. Kinematic models and simulations for trajectory planning in the cutting of Spatially-Arbitrary crosssections by a robotic roadheader[J]. Tunnelling and Underground Space Technology, 2018, 78: 115-123.

[10] Allen J C. Evolution of underground mining equipment[J]. Colo. Sch. Min. Q.;(United States), 1968, 63(4).

[11] Marshall J A, Bonchis A, Nebot E, et al. Robotics in mining[M]//Springer handbook of robotics. Springer, Cham, 2016: 1549-1576.

[12] McGarey P, Reid W, Nesnas I. Towards articulated mobility and efficient docking for the duaxel tethered robot system[C]//2019 IEEE Aerospace Conference. IEEE, 2019: 1-9.

[13] Kim B, Yi K. Probabilistic and holistic prediction of vehicle states using sensor fusion for application to integrated vehicle safety systems[J]. IEEE Transactions on Intelligent Transportation Systems, 2014, 15(5): 2178-2190.

[14] Xie J, Gong J, Wu S, et al. A personalized curve driving model for intelligent vehicle[C]//2017 IEEE International Conference on Unmanned Systems (ICUS). IEEE, 2017: 301-306.

[15] Owens B. Concept Design and Testing of a GPS-less System for Autonomous Shovel-Truck Spotting[D]. , 2013.

[16] Nayl T, Nikolakopoulos G, Gustfsson T. Switching model predictive control for an articulated vehicle under varying slip angle[C]//2012 20th Mediterranean Conference on Control \& Automation (MED). IEEE, 2012: 890-895.

[17] Martínez J L, Mandow A, Morales J, et al. Approximating kinematics for tracked mobile robots[J]. The International Journal of Robotics Research, 2005, 24(10): 867-878.

[18] Abbaspour H, Drebenstedt C, Dindarloo S R. Evaluation of safety and social indexes in the selection of transportation system alternatives (Truck-Shovel and IPCCs) in open pit mines[J]. Safety science, 2018, 108: 1-12.

[19] Chen S L, Wu K C. Contouring control of smooth paths for multiaxis motion systems based on equivalent errors[J]. IEEE Transactions on Control Systems Technology, 2007, 15(6): 1151-1158.

[20] Angelov P, Yager R. A new type of simplified fuzzy rule-based system[J]. International Journal of General Systems, 2012, 41(2): 163-185.

[21] Lei K, Shao Y, Zhang Y, et al. Research on control method of three-phase inverter based on LQR optimal tracking control[C]//Journal of Physics: Conference Series. IOP Publishing, 2021, 1871(1): 012061.

[22] Neacşu D O, Sîrbu A. Design of a LQR-based boost converter controller for energy savings[J]. IEEE Transactions on Industrial Electronics, 2019, 67(7): 5379-5388.

[23] Kumar E V, Jerome J. Robust LQR controller design for stabilizing and trajectory tracking of inverted pendulum[J]. Procedia Engineering, 2013, 64: 169-178.

[24] Hinrichsen D, Pritchard A J. Mathematical systems theory I: modelling, state space analysis, stability and robustness[M]. Springer Science \& Business Media, 2011. 
[25] Feng C, Dong S, Lundeen K M, et al. Vision-based articulated machine pose estimation for excavation monitoring and guidance[C]//ISARC. Proceedings of the International Symposium on Automation and Robotics in Construction. IAARC Publications, 2015, 32: 1.

[26] Dai Y, Song J, Yu L. Vehicle Stability Control on Tire Burst Steering and Braking Condition With Active Steering System[C]//ASME 2018 International Design Engineering Technical Conferences and Computers and Information in Engineering Conference. American Society of Mechanical Engineers Digital Collection, 2018.

[27] Rane A K, Kumar S, Maheshwari S. Literature Review on Analysis of Wheel Loader and Its Various Components[J]. Materials Today: Proceedings, 2018, 5(9): 19049-19055.

[28] Kilmer M E, Braman K, Hao N, et al. Third-order tensors as operators on matrices: A theoretical and computational framework with applications in imaging[J]. SIAM Journal on Matrix Analysis and Applications, 2013, 34(1): 148-172.

[29] Shekhar R C, Maciejowski J M. Surface excavation with model predictive control[C]//49th IEEE Conference on Decision and Control (CDC). IEEE, 2010: 5239-5244.

[30] Atanasov N, Le Ny J, Daniilidis K, et al. Decentralized active information acquisition: Theory and application to multi-robot SLAM[C]//2015 IEEE International Conference on Robotics and Automation (ICRA). IEEE, 2015: 4775-4782.

[31] Wood S N. Fast stable restricted maximum likelihood and marginal likelihood estimation of semiparametric generalized linear models[J]. Journal of the Royal Statistical Society: Series B (Statistical Methodology), 2011, 73(1): 3-36.

[32] Kuo R J, Han Y S. A hybrid of genetic algorithm and particle swarm optimization for solving bi-level linear programming problem-A case study on supply chain model[J]. Applied Mathematical Modelling, 2011, 35(8): 3905-3917.

[33] Kamrani A K. Genetic-algorithm-based solution for combinatorial optimization problems[J]. Systems engineering tools and methods. CRC Press, Boca Raton, 2011.

[34] Garg P. A Comparison between Memetic algorithm and Genetic algorithm for the cryptanalysis of Simplified Data Encryption Standard algorithm[J]. arXiv preprint arXiv:1004.0574, 2010.

[35] Jiang F G, Wang Z Q. The truss structural optimization design based on improved hybrid genetic algorithm[C]//Advanced Materials Research. Trans Tech Publications Ltd, 2011, 163: 2304-2308.

[36] de Macêdo Braz H D, de Souza B A. Distribution network reconfiguration using genetic algorithms with sequential encoding: Subtractive and additive approaches[J]. IEEE Transactions on Power Systems, 2010, 26(2): 582-593.

[37] Roberge V, Tarbouchi M, Okou F. Strategies to accelerate harmonic minimization in multilevel inverters using a parallel genetic algorithm on graphical processing unit[J]. IEEE transactions on power electronics, 2014, 29(10): 50875090 .

[38] Wang J, Liang K, Huang X, et al. Dissipative fault-tolerant control for nonlinear singular perturbed systems with Markov jumping parameters based on slow state feedback[J]. Applied Mathematics and Computation, 2018, 328: 247262.

[39] Arqub O A, Abo-Hammour Z. Numerical solution of systems of second-order boundary value problems using continuous genetic algorithm[J]. Information sciences, 2014, 279: 396-415.

[40] Oberle W. Monte Carlo simulations: number of iterations and accuracy[R]. ARMY RESEARCH LAB ABERDEEN PROVING GROUND MD WEAPONS AND MATERIALS RESEARCH DIRECTORATE, 2015. 
[41] Li B, Tian H, Jing Q, et al. Innovative LQR Control Method for Vessel Rudder Control[C]//2019 Chinese Control Conference (CCC). IEEE, 2019: 3559-3563.

[42] Shi J, Sun D, Qin D, et al. Planning the trajectory of an autonomous wheel loader and tracking its trajectory via adaptive model predictive control[J]. Robotics and Autonomous Systems, 2020, 131: 103570.

[43] Shariati H, Yeraliyev A, Terai B, et al. Towards autonomous mining via intelligent excavators[C]//Proceedings of the IEEE/CVF Conference on Computer Vision and Pattern Recognition Workshops. 2019: 26-32.

[44] Modares H, Alfi A, Fateh M M. Parameter identification of chaotic dynamic systems through an improved particle swarm optimization[J]. Expert Systems with Applications, 2010, 37(5): 3714-3720.

[45]Lee C P, Lin W S, Chen Y M, et al. Gene selection and sample classification on microarray data based on adaptive genetic algorithm/k-nearest neighbor method[J]. Expert Systems with Applications, 2011, 38(5): 4661-4667.

[46]Liu H, Cai Z, Wang Y. Hybridizing particle swarm optimization with differential evolution for constrained numerical and engineering optimization[J]. Applied Soft Computing, 2010, 10(2): 629-640.

[47] Klidbary S H, Shouraki S B, Kourabbaslou S S. Path planning of modular robots on various terrains using Q-learning versus optimization algorithms[J]. Intelligent Service Robotics, 2017, 10(2): 121-136.

[48] Fang J. The LQR controller design of two-wheeled self-balancing robot based on the particle swarm optimization algorithm[J]. Mathematical Problems in Engineering, 2014, 2014.

[49] Sun J, Fang W, Wu X, et al. Quantum-behaved particle swarm optimization: analysis of individual particle behavior and parameter selection[J]. Evolutionary computation, 2012, 20(3): 349-393.

[50] Yi L. Study on an improved PSO algorithm and its application for solving function problem[J]. Int. J. Smart Home, 2016, 10(3): 51-62.

[51]Ghimatgar H, Kazemi K, Helfroush M S, et al. An improved feature selection algorithm based on graph clustering and ant colony optimization[J]. Knowledge-Based Systems, 2018, 159: 270-285.

[52]Zhang Y, Wang S, Ji G. A rule-based model for bankruptcy prediction based on an improved genetic ant colony algorithm[J]. Mathematical Problems in Engineering, 2013, 2013.

[53] Lawhern V, Hairston W D, Robbins K. DETECT: A MATLAB toolbox for event detection and identification in time series, with applications to artifact detection in EEG signals[J]. PloS one, 2013, 8(4): e62944.

[54] Duan P, Yong A I. Research on an improved ant colony optimization algorithm and its application[J]. International Journal of Hybrid Information Technology, 2016, 9(4): 223-234.

[55] Saidi-Mehrabad M, Dehnavi-Arani S, Evazabadian F, et al. An Ant Colony Algorithm (ACA) for solving the new integrated model of job shop scheduling and conflict-free routing of AGVs[J]. Computers \& Industrial Engineering, 2015, 86: 2-13. 